\title{
The Extinction Properties of and Distance to the Highly Reddened Type IA Supernova 2012cu
}

X. Huang ${ }^{1}$, Z. Raha ${ }^{1}$, G. Aldering ${ }^{2}$, P. Antilogus ${ }^{3}$, S. Bailey ${ }^{2}$, C. Baltay ${ }^{4}$, K. Barbary ${ }^{5}$, D. Baugh ${ }^{6}$, K. Boone ${ }^{2,5}$, S. Bongard ${ }^{3}$,

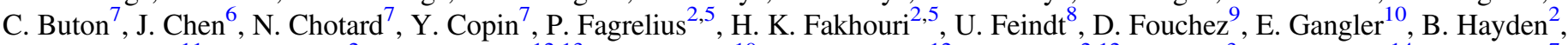
W. Hillebrandt ${ }^{11}$, A. G. Kim ${ }^{2}$, M. Kowalski ${ }^{12,13}$, P.-F. Leget ${ }^{10}$, S. Lombardo ${ }^{12}$, J. Nordin ${ }^{2,12}$, R. Pain ${ }^{3}$, E. Pecontal ${ }^{14}$, R. Pereira ${ }^{7}$, S. Perlmutter ${ }^{2,5}$, D. Rabinowitz ${ }^{4}$, M. Rigault ${ }^{12}$, D. Rubin ${ }^{2,15}$, K. Runge ${ }^{2}$, C. Saunders ${ }^{2,5}$, G. Smadja ${ }^{7}$, C. Sofiatti ${ }^{2,5}$, A. Stocker ${ }^{16}$, N. Suzuki ${ }^{2,17}$, S. Taubenberger ${ }^{11}$, C. Tao ${ }^{6,9}$, and R. C. Thomas ${ }^{18}$

(The Nearby Supernova Factory)

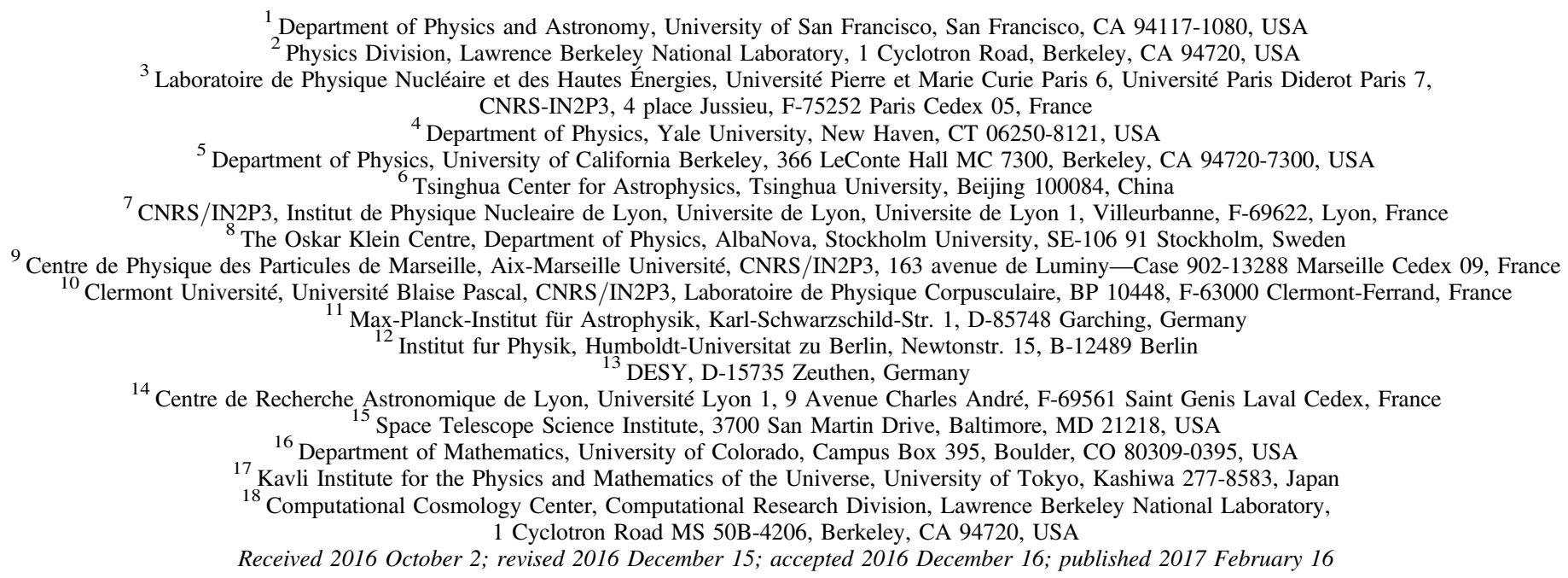

\begin{abstract}
Correcting Type Ia Supernova brightnesses for extinction by dust has proven to be a vexing problem. Here we study the dust foreground to the highly reddened SN 2012cu, which is projected onto a dust lane in the galaxy NGC 4772. The analysis is based on multi-epoch, spectrophotometric observations spanning from 3300-9200 A, obtained by the Nearby Supernova Factory. Phase-matched comparison of the spectroscopically twinned SN $2012 \mathrm{cu}$ and SN 2011fe across 10 epochs results in the best-fit color excess of $(E(B-V)$, RMS $)=(1.00,0.03)$ and total-to-selective extinction ratio of $\left(R_{V}, \mathrm{RMS}\right)=(2.95,0.08)$ toward SN 2012cu within its host galaxy. We further identify several diffuse interstellar bands and compare the $5780 \AA$ band with the dust-to-band ratio for the Milky Way (MW). Overall, we find the foreground dust-extinction properties for SN 2012cu to be consistent with those of the MW. Furthermore, we find no evidence for significant time variation in any of these extinction tracers. We also compare the dust extinction curve models of Cardelli et al., O'Donnell, and Fitzpatrick, and find the predictions of Fitzpatrick fit SN 2012cu the best. Finally, the distance to NGC4772, the host of SN 2012cu, at a redshift of $z=0.0035$, often assigned to the Virgo Southern Extension, is determined to be $16.6 \pm 1.1 \mathrm{Mpc}$. We compare this result with distance measurements in the literature.
\end{abstract}

Key words: cosmology: observations - distance scale - dust, extinction - supernovae: individual (SN 2012cu)

\section{Introduction}

The distance measurements of Type Ia supernovae (SNe Ia) led to the discovery of the accelerated expansion of the universe (Riess et al. 1998; Perlmutter et al. 1999). This technique, based on the standardizability of the peak luminosity of SNe Ia, remains one of the most powerful probes for understanding the cause of the acceleration (e.g., Suzuki et al. 2012; Betoule et al. 2014; Rest et al. 2014). This is especially true in light of recent developments in standardization techniques that promise to substantially reduce brightness dispersion from the canonical value of $\sim 0.15 \mathrm{mag}$, alternatively based on the incorporation of near-IR light curves, Si II $\lambda 6355 \AA$ absorption line velocity, and local star formation rate (Wang et al. 2009; Foley \& Kasen 2011; Mandel et al. 2011; Barone-Nugent et al. 2012; Rigault et al. 2013; Kelly et al. 2015). Recently, Fakhouri et al. (2015) proposed a new standardization procedure based on a spectroscopic twinning method in the optical range that produces a dispersion that is as good as the best of other standardization methods. With the prospect that the variation due to the astrophysical differences of SNe Ia can be further minimized with the twinning method, dust remains one of the last key sources of systematic uncertainty.

Following Cardelli et al. (1989), dust extinction properties are often characterized by one parameter, $R_{V} \equiv A_{V} / E(B-V)$, the total-to-selective extinction ratio. The interstellar dust in the 
Milky Way (MW) has a well-known average $R_{V}$ of 3.1 (e.g., Draine 2003; Schlafly \& Finkbeiner 2011). The slope of the color standardization for $\mathrm{SNe}$ Ia is determined by minimizing the rest-frame $B$-band luminosity $\left(M_{B}\right)$ scatter (e.g., Tripp 1998; Tripp \& Branch 1999). The slope, usually denoted as $\beta$ (e.g., Guy et al. 2010, Equation (5)), could naively be expected to equal $R_{B}\left(=R_{V}+1\right)$, but likely mixes the effects of $\mathrm{SN}$ intrinsic color variation and dust. In fact, typical fits for $\beta$ yield much lower values (e.g., Tripp 1998; Hicken et al. 2009; Wang et al. 2009) than expected given the average MW $R_{V}$.

Chotard et al. (2011) shed light on this apparent discrepancy. Exploiting the fact that spectral features are independent of dust extinction, they first showed that, by applying corrections based on the equivalent widths (EWs) of the Si II $\lambda 4131 \AA$ and Ca II H\&K features, two highly variable regions in the SN spectra, the mean empirical extinction law obtained by minimizing $M_{B}$ scatter for their SN sample became much smoother and conformed well to the extinction law of Cardelli et al. (1989, CCM89). Second, by adopting a color covariance matrix that is different from the one based on the output of the SALT2 light curve fitter (Guy et al. 2007), they obtained $R_{V}=2.8 \pm 0.3$, in agreement with the average MW value. It remains the case, however, that many highly reddened SNe tend to have an $R_{V}$ lower than 2 (e.g., Mandel et al. 2011; Phillips et al. 2013). A recent example is SN 2014J, a wellobserved, highly reddened SN Ia with an $R_{V}$ of $1.4-1.7$ and $E(B-V)$ of 1.2-1.4 (Amanullah et al. 2014; Foley et al. 2014; Goobar et al. 2014; Brown et al. 2015; Marion et al. 2015). These results are not derived from brightness-color behavior, as with the $\beta$ parameter mentioned above, but rather, are obtained from a fit of the shape of the SN spectral energy distribution using an extinction curve parameterized by $R_{V}$.

Unlike for cosmological studies, in order to understand the extinction properties of host galaxies, highly reddened $\mathrm{SNe}$ are useful. Comparing a reddened SN Ia with its spectroscopic twin that is little affected by dust would allow the study of hostgalaxy extinction without the confusion of the intrinsic color variation. SN 2012cu presents such an opportunity, as it has one of the highest extinction values observed to date. Furthermore, SN 2011fe, in many regards the best studied SN Ia, is a spectroscopic twin of SN 2012cu. The fact that SN 2011fe has little or no dust (Nugent et al. 2011) is also quite important, since the comparison of SN 2012cu will provide the total-not just differential-extinction. In this paper, with SN 2011fe as the template, we analyze the wavelengthdependent extinction of SN 2012cu using a spectrophotometric time series obtained by the Nearby Supernova Factory (SNfactory, Aldering et al. 2002). While the nearly ideal pairing between SN 2012cu and SN 2011fe is perhaps rare today, such samples should continue to grow. The $R_{V}$ distribution found nearby will then inform high-redshift studies, just as light curve parameters now do.

With SN 2012cu dereddened, we further demonstrate that in the case of SN 2012cu and SN 2011fe, the twinning approach can provide highly precise and accurate relative distance measurements, as Fakhouri et al. (2015) found.

This paper is organized as follows. We present our spectroscopic observations of SN 2012cu in Section 2. In Section 3, we measure $E(B-V), R_{V}, A_{V}$, and the relative distance between SN 2012cu and SN 2011fe, using data from 10 epochs between -6.8 days and 23.2 days measured relative to the DayMax parameter of the SALT light curve fitter (Guy et al. 2007, 2010). We explore different approaches for handling the spectral mismatches that remain. We also measure the EWs of $\mathrm{Na}$ I and a diffuse interstellar band (DIB) feature at $5780 \AA$ and examine whether these features exhibit time variability. In Section 4, we determine the distance of SN 2012cu to the host galaxy and discuss the nature of foreground dust in its host. We present our conclusions in Section 5 .

\section{Observations}

SN 2012cu was discovered on 2012 June 11.2 UT (Ganeshalingam et al. 2012; Itagaki et al. 2012) and was classified as a Type Ia on 2012 June 15, UT (Marion et al. 2012; Zhang et al. 2012). NGC 4772 is the host, at a heliocentric redshift of $z=0.003469 \pm 0.000017$ (de Vaucouleurs et al. 1991).

Figure 1 shows our $V$-band image of SN 2012cu and the host galaxy, NGC 4772. The upper left inset is an image from the Wide-field Infrared Survey Explorer (WISE, Wright et al. 2010) in the $12 \mu \mathrm{m}$ band, which spans the wavelengths of some of the emission features of polycyclic aromatic hydrocarbons associated with dust (Li \& Draine 2001). The upper right inset is a GALEX (Bianchi et al. 2014) image that highlights the young stars along the dust lane. The comparison of these images shows that $\mathrm{SN} 2012 \mathrm{cu}$ is projected onto a dust ring. There is a reasonably high probability that $\mathrm{SN} 2012 \mathrm{cu}$ is behind or embedded in the dust lane, and that it is mostly reddened by this dust detected in the interstellar medium (ISM) of the host galaxy.

Spectra of SN 2012cu, spanning 17 epochs from -6.8 days to 46.2 days were obtained by the SNfactory collaboration with its SuperNova Integral Field Spectrograph (SNIFS; Aldering et al. 2002; Lantz et al. 2004) on the University of Hawaii $2.2 \mathrm{~m}$ telescope on Maunakea. For the wavelength range of 3300-9200 $\AA$, the spectra of SN 2012cu are flux calibrated (Buton et al. 2013), host-galaxy subtracted (Bongard et al. 2011), corrected for MW extinction (Schlafly \& Finkbeiner 2011), and then deredshifted to the rest frame for subsequent analysis. Further details, including the observing $\log$, a figure showing the entire the spectral time series, and information on how to access these data are provided in Appendix B.

\section{Dust Extinction Properties}

In this section, we first describe how we fit for the dust extinction curve and the distance modulus difference $(\Delta \mu)$ between SN 2012cu and SN 2011fe (Section 3.1). In this effort, we explore different deweighting schemes for regions where spectral differences are present. We then turn our attention to Na I and DIB features in the SN 2012cu spectra (Section 3.2). Dust extinction curves are parameterized by quantities denoted as $E(B-V)$ and $R_{V}$; these are not required to match the values that would be measured only using the $B$ and $V$ bands.

\subsection{Extinction Curve and Distance Modulus Difference}

3.1.1. Simultaneously Fitting for $E(B-V), R_{V}$, and $\Delta \mu$

The best-fit SALT2.4 parameters for SN 2012cu are first determined to be $\left(x_{1}^{\prime}, c^{\prime}\right)=(0.021 \pm 0.123,0.971 \pm 0.030)$ and DayMax $^{\prime}=$ MJD 56104.89 \pm 0.15 . Given the extreme 

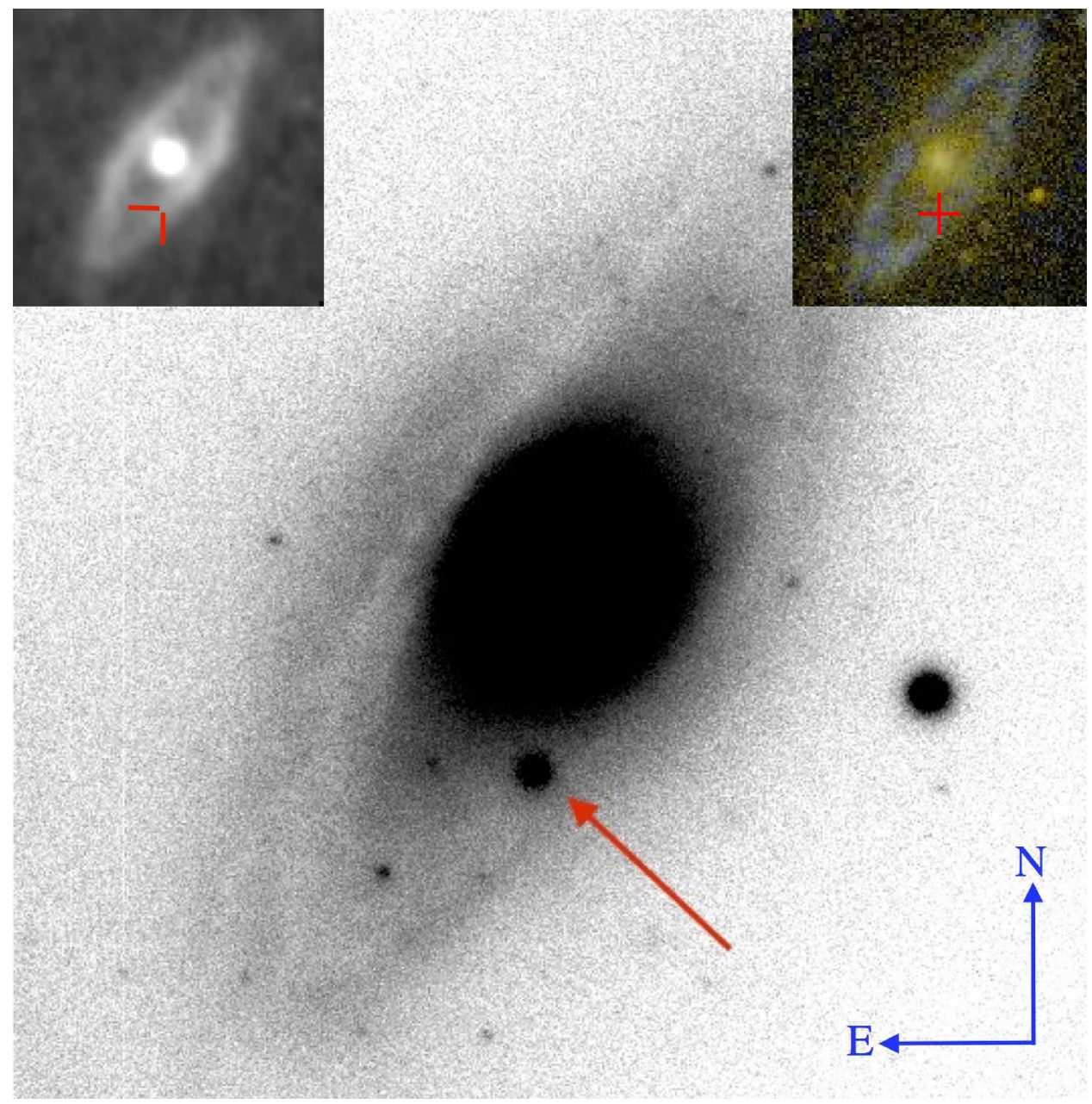

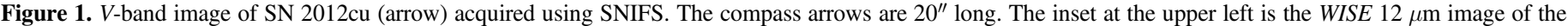

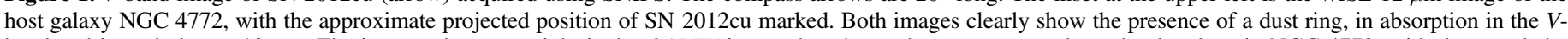

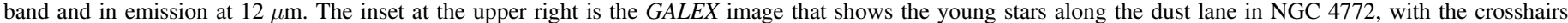
marking the position of SN $2012 \mathrm{cu}$.

color of SN 2012cu, much higher than the typical reddening in the training data for SALT, we apply an initial dereddening using the extinction curve of Fitzpatrick (1999) and perform the fit again. We find $x_{1}=-0.269 \pm 0.078$ and DayMax $=$ MJD 56105.06 \pm 0.11 . The DayMax value has shifted by +0.16 days, and corresponds to June 27.1 UT. To be consistent, we perform the SALT2.4 fit for SN 2011fe as well, and obtain light curve parameters $\left(x_{1}, c\right)=(-0.280 \pm 0.093$, $-0.058 \pm 0.027$ ) and DayMax $=55815.20 \pm 0.08$. Our $x_{1}, c$, and DayMax are in good agreement with the corresponding values in Pereira et al. 2013, using SALT2.2. The $x_{1}$ parameter is now very similar for these two SNe. We further determine that this pair has a near-maximum twinness ranking of 0.1 , on a scale from 0 to 1 , placing the pairing of these two SNe among the best spectroscopic "twins" (Fakhouri et al. 2015, Figure 5). It is even the case that both $\mathrm{SNe}$ exhibit the rare $\mathrm{C}$ II feature (Thomas et al. 2011), albeit at higher velocity for SN 2012cu at the earliest phase, strengthening the case for the similarity of the explosion physics.

As SN 2011fe suffers from nearly no host extinction, for the rest of this paper we treat its spectra, which have been corrected for mild MW extinction $(E(B-V)=0.0088 \mathrm{mag})$ by Pereira et al. (2013), as if they were unreddened versions of SN 2012cu spectra.
To determine the effects of dust reddening for SN 2012cu, we first pair the SN $2011 \mathrm{fe}$ spectra, also obtained with SNIFS (Pereira et al. 2013), with those of SN 2012cu for cases where the phase difference is less than one day. This is possible for 10 SN 2012cu phases between -6.8 days and 23.2 days, thanks to the dense temporal sampling of the Pereira et al. (2013) data set (see Table 1). In this paper, the phases for both $\mathrm{SNe}$ are always measured relative to their respective DayMax. The phase differences (0.2-0.3 days; see Table 1) are somewhat larger than the combined phase uncertainty between the two SNe ( 0.14 days). As a test, we have performed interpolation for several phases, including for phase pairings with a 0.3 day phase difference, and find these phase differences make very little difference for the best-fit results. Thus, there is no need to interpolate.

For these 10 pairs of spectra, we divide the fluxes evenly into 925 bins above $3600 \AA$; between 3300 and $3600 \AA$ we use two bins due to the lower signal-to-noise ratio $(\mathrm{S} / \mathrm{N})$ for the highly reddened SN 2012cu. Therefore, there are a total of 927 bins. We use these same bins for both SNe.

The extinction curve models of Cardelli et al. (1989), O'Donnell (1994), and Fitzpatrick (1999) (CCM89, OD94, and F99, respectively) are the most commonly used in the literature and all three are parameterized by $R_{V}$ and $E(B-V)$. 

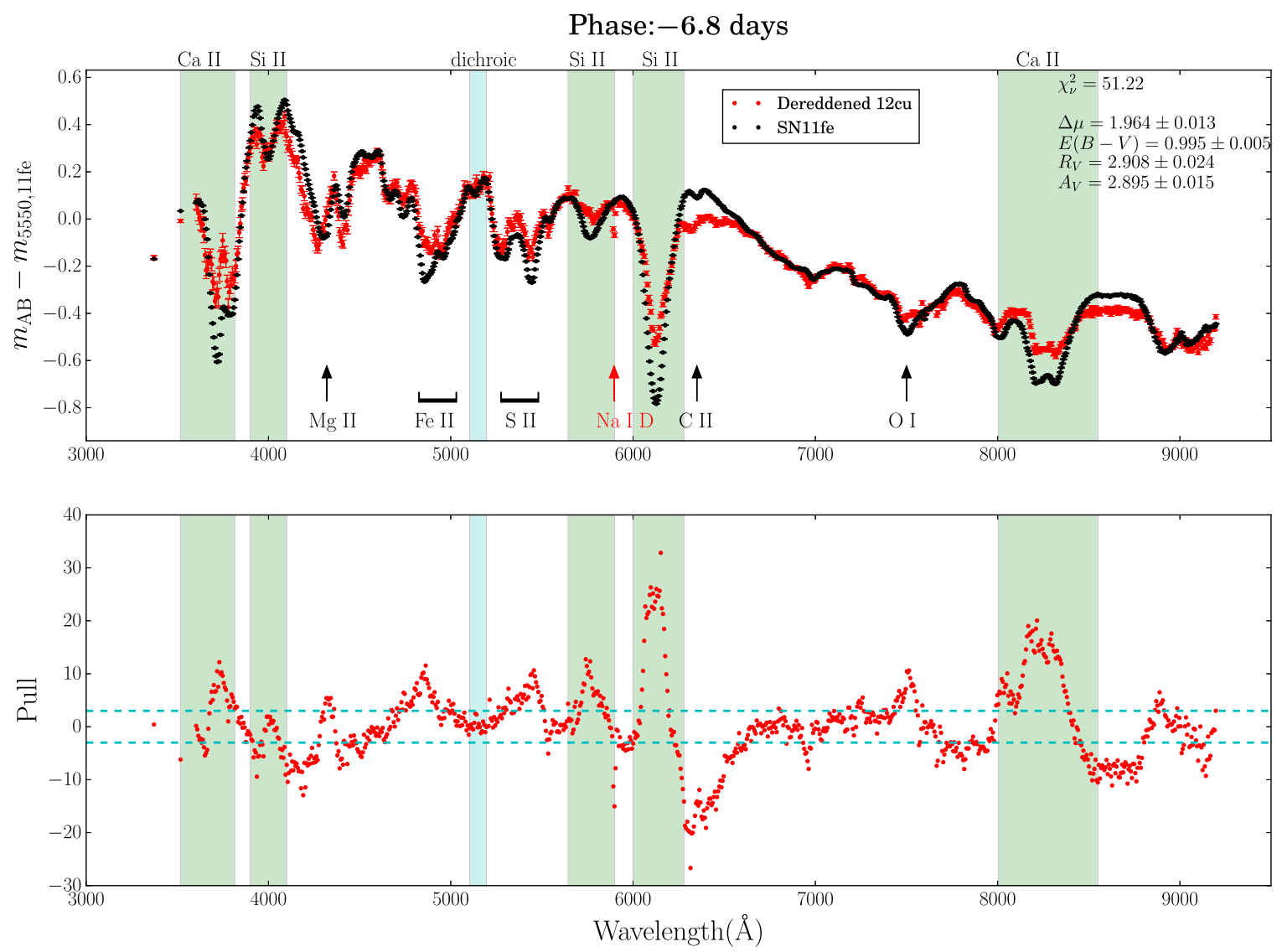

Figure 2. The top panel shows the binned SN 2012cu spectrum (red) in units of AB magnitude for the phase of -6.8 days dereddened with the best-fit $E(B-V)$ and $R_{V}$, and shifted vertically by the best-fit $\Delta \mu$, for comparison with the template spectrum of SN $2011 \mathrm{fe}$ (black) for the same phase. The zero-point is arbitrary, chosen to be the magnitude at $5550 \mathrm{~A}$ for SN $2011 \mathrm{fe}$. Even for well-twinned SNe, it is for the pre-maximum phases that spectral differences are more likely, so this pair is among those with the largest level of disagreement. Very small differences can be seen here given the high $\mathrm{S} / \mathrm{N}$ of these spectra. For comparison at later phases, see Figure 4 . The vertical green bands are the regions of the Si II and Ca II features found to be highly variable between SNe in Chotard et al. (2011). Outside of these regions, some of the other well-known SN spectral features are also labeled, as well as the Na I D absorption feature. The bottom panel shows the difference in magnitude between the two spectra in the top panel (the residual), weighted by measurement uncertainty, or the "pull." The peaks and valleys correspond to where the spectra are the most different. The cyan dashed lines indicate $3 \sigma$ deviation. It is clear that the high value of $\chi_{\nu}^{2}$ is due to spectral feature differences between the two SNe, despite the fact that they are good matches relative to other SNe. While the Chotard regions capture the greatest spectral variations, the two spectra can nonetheless be very different outside of these regions.

Table 1

Phase Comparison and Best-fit Parameters Using Approach I

\begin{tabular}{|c|c|c|c|c|c|c|c|}
\hline Pair ID & $\begin{array}{l}\text { SN 2012cu } \\
\text { Phase (days) }\end{array}$ & $\begin{array}{l}\text { SN 2011fe } \\
\text { Phase (days) }\end{array}$ & $E(B-V)$ & $R_{V}$ & $\Delta \mu$ & $\begin{array}{c}A_{V} \\
\left(=R_{V} \cdot E(B-V)\right)\end{array}$ & $\chi_{\nu}^{2}$ \\
\hline 1 & -6.8 & -7.0 & $0.995 \pm 0.005$ & $2.908 \pm 0.024$ & $1.964 \pm 0.013$ & $2.895 \pm 0.015$ & 51.22 \\
\hline 2 & -1.7 & -2.0 & $0.989 \pm 0.006$ & $3.082 \pm 0.025$ & $1.873 \pm 0.012$ & $3.048 \pm 0.014$ & 88.48 \\
\hline 3 & 3.3 & 3.0 & $1.019 \pm 0.006$ & $2.987 \pm 0.024$ & $1.868 \pm 0.012$ & $3.043 \pm 0.013$ & 99.05 \\
\hline 4 & 6.3 & 6.0 & $1.017 \pm 0.006$ & $2.962 \pm 0.023$ & $1.864 \pm 0.011$ & $3.012 \pm 0.013$ & 53.41 \\
\hline 5 & 8.3 & 8.0 & $1.010 \pm 0.007$ & $2.970 \pm 0.024$ & $1.884 \pm 0.011$ & $3.001 \pm 0.012$ & 22.70 \\
\hline 6 & 11.2 & 11.0 & $0.982 \pm 0.006$ & $2.992 \pm 0.024$ & $2.043 \pm 0.010$ & $2.939 \pm 0.012$ & 20.49 \\
\hline 7 & 16.2 & 16.0 & $0.911 \pm 0.008$ & $3.218 \pm 0.033$ & $2.016 \pm 0.011$ & $2.931 \pm 0.013$ & 27.47 \\
\hline 8 & 18.2 & 18.0 & $0.907 \pm 0.009$ & $3.270 \pm 0.038$ & $1.968 \pm 0.013$ & $2.966 \pm 0.015$ & 57.26 \\
\hline 9 & 21.2 & 21.0 & $0.912 \pm 0.011$ & $3.265 \pm 0.041$ & $2.027 \pm 0.012$ & $2.976 \pm 0.014$ & 53.03 \\
\hline 10 & 23.2 & 23.0 & $0.915 \pm 0.011$ & $3.177 \pm 0.041$ & $1.998 \pm 0.012$ & $2.908 \pm 0.014$ & 26.77 \\
\hline
\end{tabular}

F99 pointed out the tension between observation and CCM89 in the wavelength range that roughly corresponds to the $i$ and $r$ bands. This has been subsequently confirmed by other authors, not just for CCM89, but also for OD94 (e.g., Schlafly et al. 2010; Mörtsell 2013). A more detailed discussion is given in Appendix A. For the remainder of this section, we will use F99.
As we will show below, despite being good "twins," the high $\mathrm{S} / \mathrm{N}$ of the SNIFS data makes the differences in spectral features between SN 2011fe and SN 2012cu clearly visible. Such differences are stronger for premaximum phases. We compare three approaches toward the regions where the spectral differences are high. In Approach I, we will treat all wavelength bins in the same manner. In Approach II, we will 


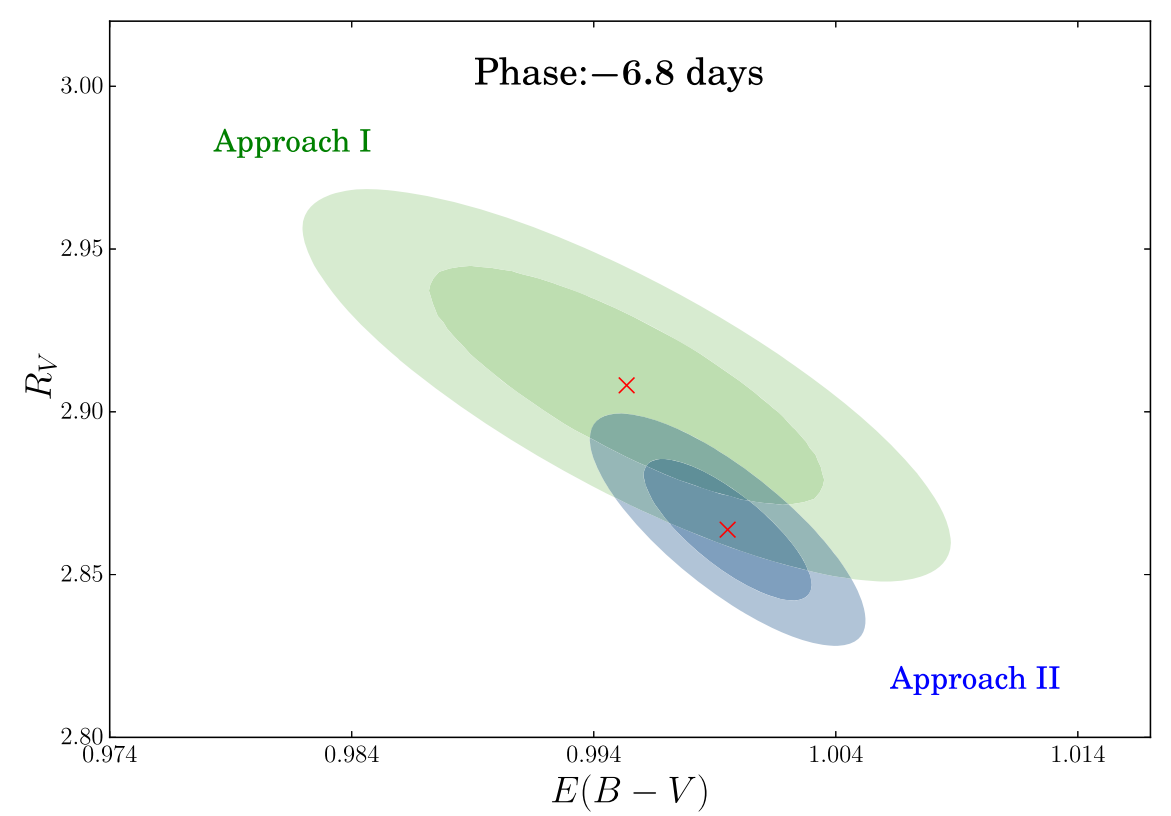

Figure 3. The green contours indicate the $1 \sigma$ and $2 \sigma$ confidence regions for $E(B-V)$ and $R_{V}$ for the phase of -6.8 days for Approach I (see the text). The cross marks the best-fit values. Likewise, the blue contours are for Approach II. All 10 phases between -6.8 days and 23.2 days exhibit a similar anti-correlation between these two fitting parameters.

identify and deweight these regions appropriately. Finally, in Approach III, we will discard these regions altogether in the fitting process.

For Approach I, when we simultaneously fit for $E(B-V)$, $R_{V}$, and $\Delta \mu$, only measurement uncertainties are included. For each phase the fit must deredden the spectrum of SN 2012cu using the dust extinction curve of F99, calculate the $\mathrm{AB}$ magnitude (see, e.g., Bessell \& Murphy 2012) for each bin, $m_{\mathrm{AB}, k}^{12 \text { dereddened }}\left(E(B-V), R_{V}\right)$, which can be more conveniently written as $m_{\mathrm{AB}, k}^{12 \mathrm{cu}}+2.5 \log \left(F 99\left(E(B-V), R_{V}\right)\right)$, and then compare the results with SN 2011fe. For each separate matching phase, we therefore minimize

$$
\begin{aligned}
& \chi^{2}=\sum_{k} \\
& \quad \frac{\left[m_{\mathrm{AB}, k}^{12 \mathrm{cu}}-m_{\mathrm{AB}, k}^{11 \mathrm{fe}}+2.5 \log \left(F 99\left(E(B-V), R_{V}\right)\right)-\Delta \mu\right]^{2}}{\sigma_{k, \text { meas }}^{2}},
\end{aligned}
$$

where $\sigma_{k \text {,meas }}^{2}=\left(\sigma_{\mathrm{AB}, k}^{11 \mathrm{fe}}\right)^{2}+\left(\sigma_{\mathrm{AB}, k}^{12 \mathrm{cu}}\right)^{2}$ is the total measurement variance for bin $k$ in magnitude space. SN 2012cu has a wavelength-independent ("gray") scatter of 0.025 mag that is uncorrelated between phases (Buton et al. 2013). For SN 2011fe, the wavelength-independent scatter is between 0.03 and $0.06 \mathrm{mag}$, again uncorrelated between phases. These larger values stem from the unusually high airmasses at which some SN $2011 \mathrm{fe}$ observations were taken. Effectively, this gray scatter is absorbed into the uncertainty for $\Delta \mu$ and does not affect $\chi^{2}$.

In Figure 2 we show the binned spectrum of SN 2012cu at phase -6.8 days in units of $\mathrm{AB}$ magnitude, dereddened with the best-fit $E(B-V)$ and $R_{V}$, and vertically shifted by the best-fit $\Delta \mu$, together with the spectrum of SN 2011fe for the corresponding phase. The minimum reduced $\chi^{2}$ is $\chi_{\nu}^{2}=51.22$. It is evident that this high value of $\chi_{\nu}^{2}$ is due to spectral feature differences between the two SNe. In the next section, we will present our main approach to address the unaccounted-for uncertainty. Here, to set a baseline for comparison with Approaches II and III, we simply uniformly inflate the errors so that $\chi_{\nu}^{2}$ is rescaled to 1 . This does not change the best-fit values or the shape of confidence contours. The error bars on the fitting parameters in the top panel are those computed after this rescaling. The vertical green bands are the regions of the highly variable Si II and Ca II features identified in Chotard et al. (2011), which will be henceforth referred to as the "Chotard regions." Outside of these regions, some of the other well-known SN spectral features are also labeled. The sharp feature around $5896 \AA$ labeled in red is Na I D absorption. The bottom panel shows the residual of the fit weighted by the measurement uncertainties, or the "pull spectrum." It is clear that the two spectra can be very different outside of the Chotard regions as well. As examples (see Figure 2) they are significantly different for: (i) the regions of Fe II $\lambda \lambda 5018,5169$, S II $\lambda 5640$, and O I $\lambda 7773$, (ii) the emission part of the P-Cygni profile of one of the Chotard Si II features, centered around $6350 \AA$, and (iii) the emission part of the Ca II IR triplet, centered around $8600 \AA$.

In Figure 3, we present the confidence contours for $E(B-V)$ and $R_{V}$, after rescaling $\chi_{\nu}^{2}$ to 1 , which is equivalent to inflating the variances in Equation (1) uniformly across all wavelengths, and marginalizing over the distance modulus difference $\Delta \mu$. $E(B-V)$ and $R_{V}$ are clearly anti-correlated; this is true for all 10 phases. The uncertainties for the extinction, $A_{V}$ (simply the product of the best-fit $E(B-V)$ and $\left.R_{V}\right)$, are computed by taking into account the covariance between these two quantities. It is notable that, owing to this anti-correlation, the uncertainty for $A_{V}$ is smaller than for that of $R_{V}$.

We apply the same fitting procedure to each of the 10 phases. The results are shown in Figure 4. Larger values of $\chi_{\nu}^{2}$ are generally associated with the earlier phases, owing to their greater spectral diversity. We will address this issue further in Section 3.1.2. The phase-by-phase summary for the best-fit $E(B-V), R_{V}, \Delta \mu$, and the derived quantity, $A_{V}$, are presented in Table 1 . The uncertainty-weighted average and RMS values across the 10 phases (Table 1), are $(E(B-V)$, 


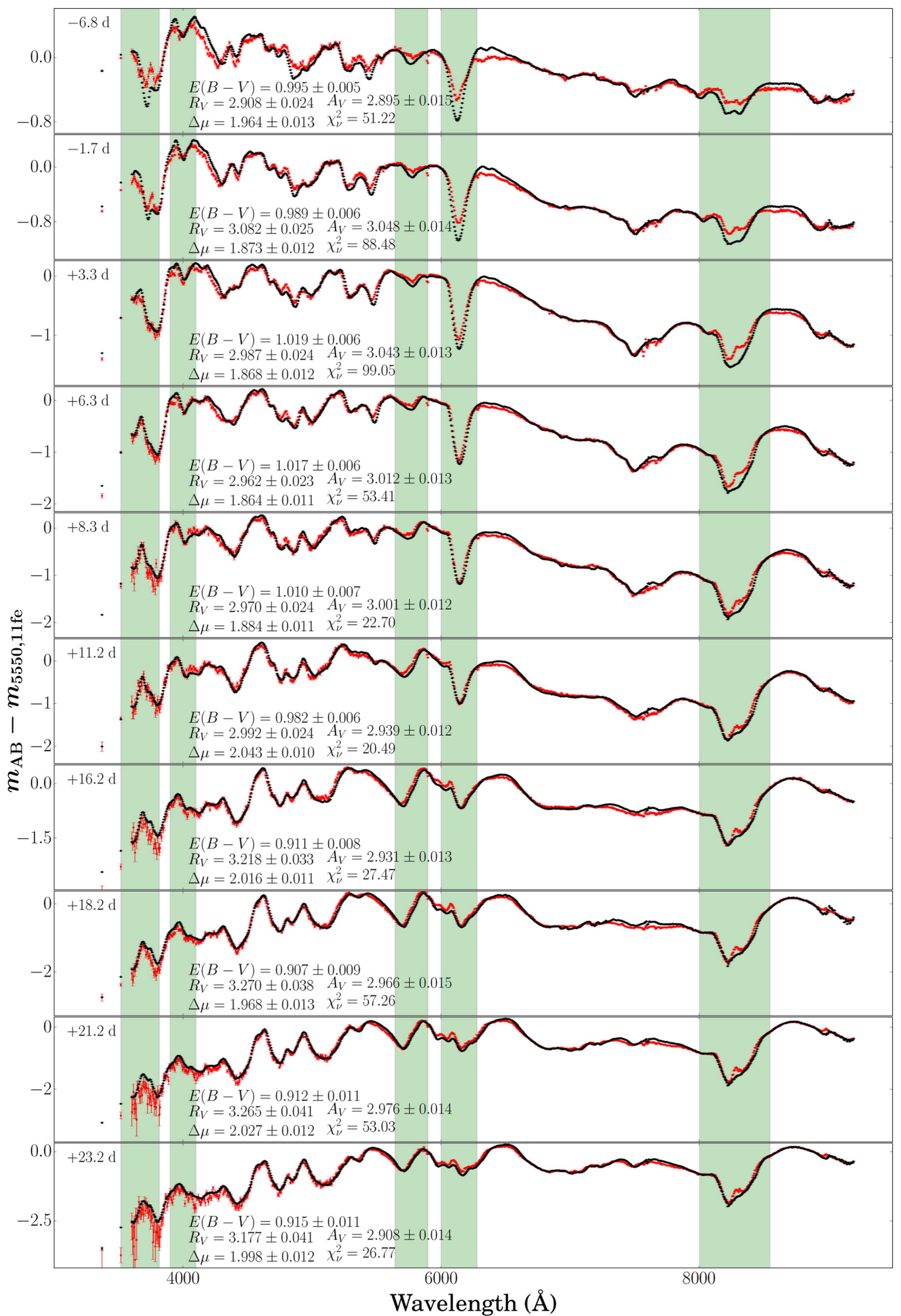

Figure 4. Full SN 2012cu spectrophotometric time series used in the analysis. The phases are shown in the upper left corner of each panel. Binned SN 2012cu spectra (red) in units of $\mathrm{AB}$ magnitude dereddened with the best-fit $E(B-V)$ and $R_{V}$, and shifted vertically by the best-fit $\Delta \mu$, are shown together with spectra of SN 2011fe with corresponding phases. As with Figure 2, the vertical green bands are the Chotard regions. 

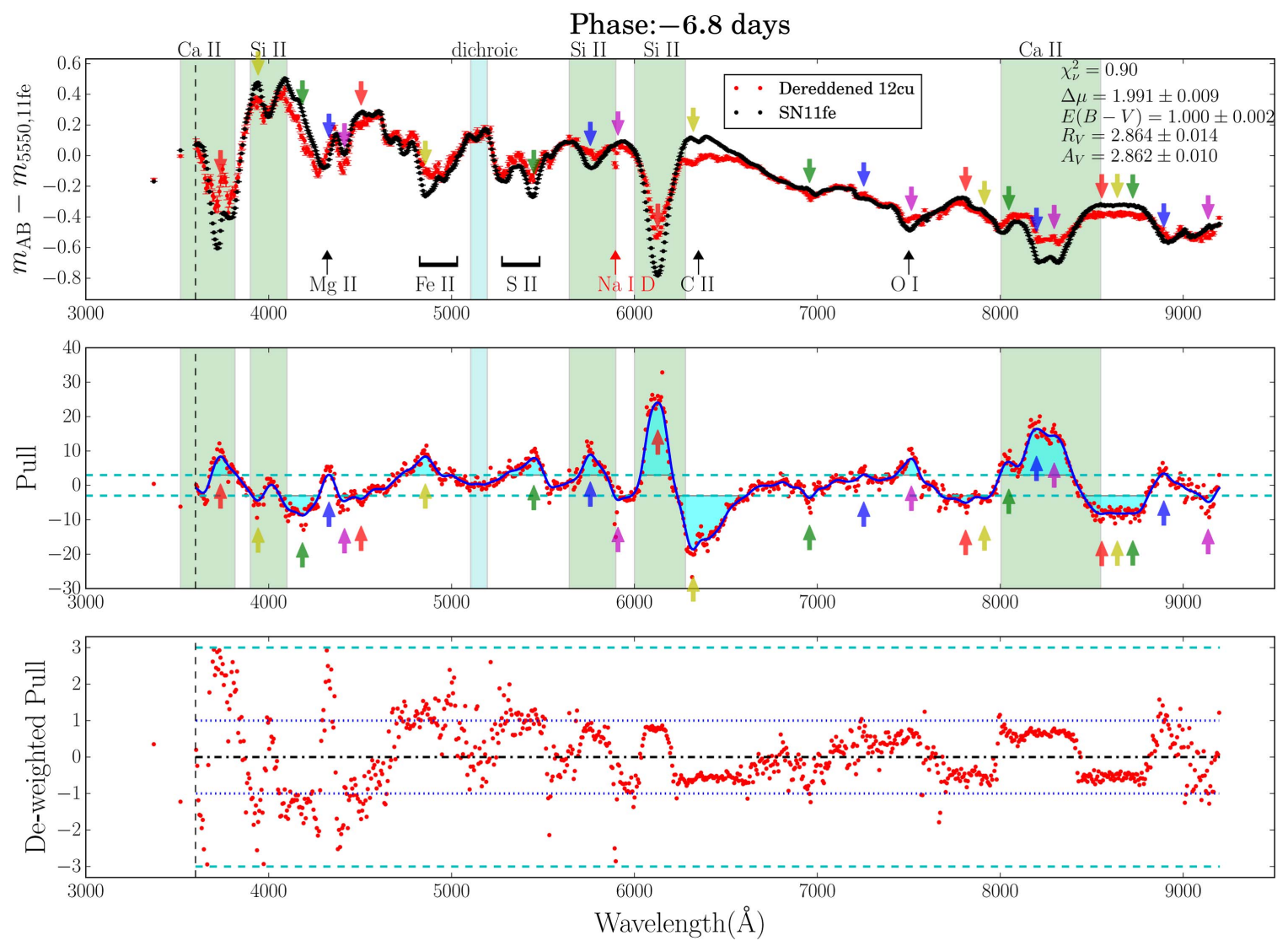

Figure 5. This figure demonstrates the technique used in Approach II to deweight the regions where the spectral differences between SN 2011fe and SN2012cu are large. Top panel: the best-fit result after our technique has been applied, achieving $\chi_{\nu}^{2} \sim 1$ as expected. Middle panel: this panel demonstrates the deweighting used in this technique. The pull spectrum shown here is the same as the one in the middle panel of Figure 2, i.e., before deweighting. The solid blue line is the result of the Gaussian convolution (see the text). Each arrow points to an extremum whose absolute value is greater than $3 \sigma$, marked by the cyan dashed lines. The arrows are matched by color between the top and middle panels and demonstrate that spectral feature differences are identified by this technique. The filled cyan regions are the wavelength regions where the deviation is greater than $3 \sigma$. To deweight these regions, an appropriately scaled quantity, $p_{k}$ (see the text), is added in quadrature to the measurement uncertainty in Equation (1) so that the $\chi_{\nu}^{2}$ for these cyan regions is 1 . In addition, we add a 0.03 mag error floor to the complementary wavelength regions, where the deviation is $\leqslant 3 \sigma$, to achieve an average of $\chi_{\nu}^{2} \sim 1$ across all phases (see top panel). Bottom panel: the pull spectrum after the application of our technique (i.e., after deweighting). The blue dotted and cyan dashed lines indicate $1 \sigma$ and $3 \sigma$ deviations, respectively.

$\mathrm{RMS})=(0.983,0.046) \mathrm{mag},\left(R_{V}, \mathrm{RMS}\right)=(3.035,0.131)$ $(\Delta \mu, \mathrm{RMS})=(1.952,0.068) \mathrm{mag}$, and $\left(A_{V}, \mathrm{RMS}\right)=(2.973$, $0.051)$ mag. Conservatively, for $R_{V}$ and $E(B-V)$, we take the uncertainties on the means to be the RMS dispersions. The uncertainty on $\Delta \mu$ is discussed in Section 4.1 , as it requires further consideration.

\subsubsection{Effects of Spectral Feature Differences}

In the previous section we showed that even though $\mathrm{SN}$ 2012cu and SN 2011fe are good "twins," there are significant spectral differences between them. In this section, by presenting the results of two alternative ways of handling these spectral feature differences, we will show that they do not have a significant effect on the best-fit extinction and relative distance parameters.

In Approach II, we deweight SN spectral feature differences but leave the differences due to measurement noise intact. To identify where the spectra behave differently for SN 2011fe and SN 2012cu, the terms in the summation in Equation (1) are plotted against the central wavelengths of the corresponding bins. As shown in Figure 2, in the (residual $/ \sigma$ ) spectrum, or pull spectrum, the locations of the peaks and valleys are where the spectra of 2011fe and 2012cu are the most different. We convolve the pull spectrum with a Gaussian kernel and through trial and error find that a Gaussian with a standard deviation of $25 \AA$ captures the spectrally correlated differences (features) discernible by eye (Figure 5). We now use the result of the convolution, $p_{k}$, to deweight the spectral differences. First we set $p_{k}$ to zero for the wavelength bins where $\left|p_{k}\right| \leqslant 3$. We deweight the complementary bins, where $\left|p_{k}\right|>3$, by rescaling $p_{k}$ in such a way that when added in quadrature to the denominator in Equation (1), the reduced $\chi^{2}$ for these regions is 1. The spectral regions that are not deweighted span the full wavelength range. We then add the rescaled $p_{k}^{2}$ to $\sigma_{k \text {, meas }}^{2}$ in the denominator of Equation (1). In order to achieve an average $\chi_{\nu}^{2} \sim 1$ across all phases, we find that for the wavelength regions where $\left|p_{k}\right| \leqslant 3$, it is necessary to add an error floor of $\sigma_{\text {floor }}=0.03 \mathrm{mag}$. This error floor is treated as an uncorrelated error, and is not related to the gray scatter mentioned in Section 3.1.1. Thus, Equation (1) becomes

$$
=\sum_{k} \frac{\left[m_{\mathrm{AB}, k}^{12 \mathrm{cu}}-m_{\mathrm{AB}, k}^{11 \mathrm{fe}}+2.5 \log \left(F 99\left(E(B-V), R_{V}\right)\right)+\Delta \mu\right]^{2}}{\sigma_{k \text {,meas }}^{2}+p_{k}^{2}+\sigma_{\text {floor }}^{2}}
$$

Using Equation (2), we once more perform $\chi^{2}$ minimization In Figure 5 we show the best-fit dereddened SN 2012cu 

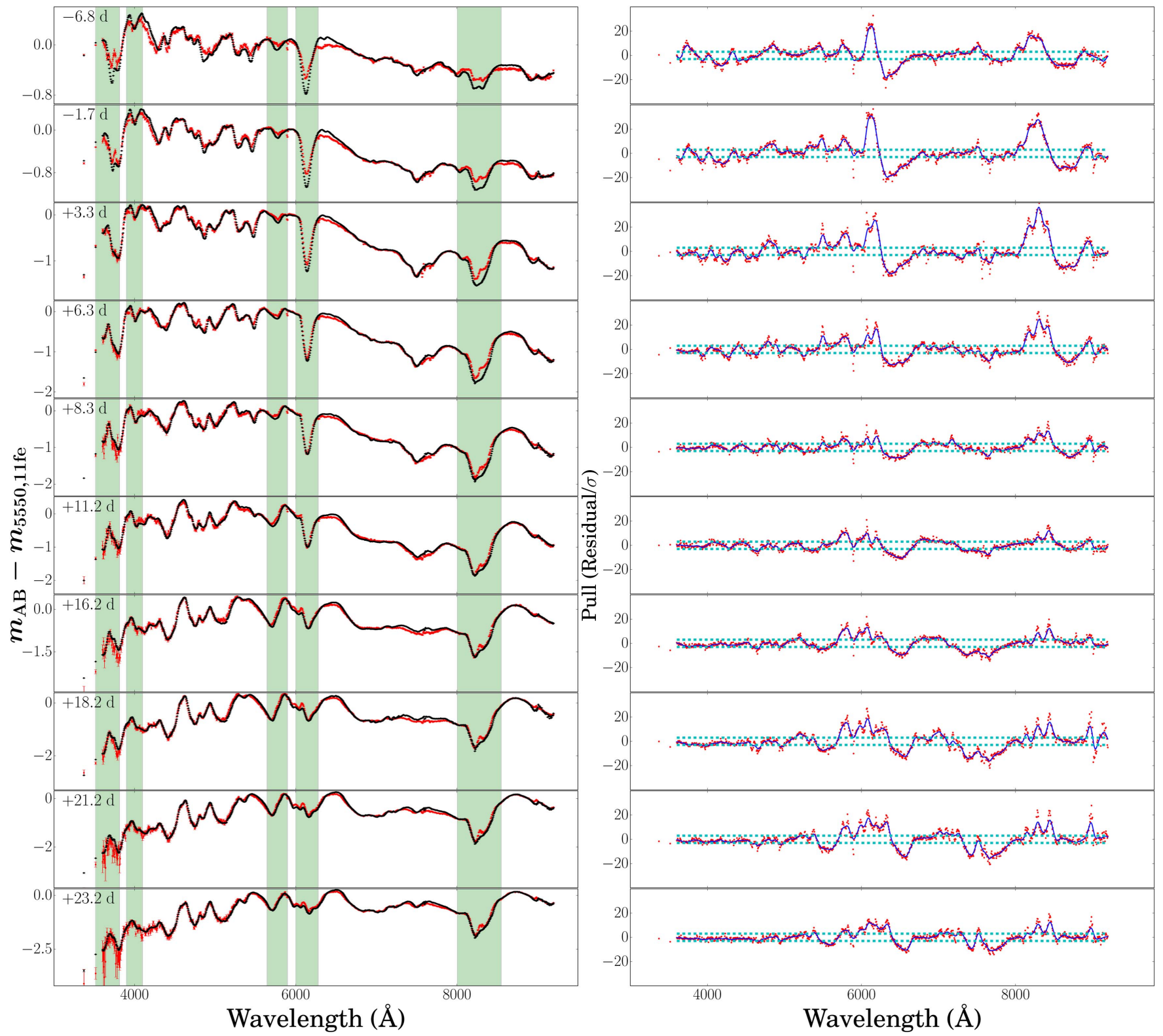

Figure 6. The left panels are the same as Figure 4, except the fitting process now is that of Approach II, which deweights the wavelength regions where there are significant spectral feature differences (see the text). The results of convolving the pull spectrum for each phase with a Gaussian kernel are shown in the right panels. The cyan dashed lines indicate $3 \sigma$ deviations.

spectrum at -6.8 days, vertically shifted by the best-fit $\Delta \mu$, together with the $2011 \mathrm{fe}$ spectrum at the corresponding phase. We obtain $\chi_{\nu}^{2}=0.90$, which is close to 1 , as expected. We note the pull values (third panel in Figure 5) for $\lambda \lesssim 6000 \AA$ are slightly larger than those for the longer wavelengths because the measurement uncertainties for the bluer wavelengths are larger due to extinction and therefore a uniform error floor does not have as much of an effect. However, we will show below that the particular choice of a weighting scheme does not affect the best-fit results in a significant way. We then apply the same fitting procedure to each of the 10 phases. The results are presented in the left panels of Figure 6 and Table 2. The values of $\chi_{\nu}^{2}$ now range from 0.78 to 1.24 . The right panels of Figure 6 show the convolution result for the pull spectrum for each of the 10 epochs.
The weighted average and RMS values across the 10 phases are $(E(B-V), \mathrm{RMS})=(1.002,0.031),\left(R_{V}, \mathrm{RMS}\right)=(2.952$, $0.081),(\Delta \mu, \mathrm{RMS})=(1.982,0.061)$, and $\left(A_{V}, \mathrm{RMS}\right)=(2.944$, 0.043). These results, along with those from Approach I, are summarized in Table 3 and Figure 7. Once again we take the uncertainties on the means for $R_{V}$ and $E(B-V)$ to be the RMS dispersions across phases.

We have pursued one more approach. In Approach III, we increase $p_{k}$ to an arbitrarily high value, essentially removing data in the wavelength regions with pull values greater than 3 (the cyan regions in Figure 5) from the fitting process. The average values of our best-fit parameters across the 10 epochs are $(E(B-V), \mathrm{RMS})=(0.999,0.029),\left(R_{V}, \mathrm{RMS}\right)=(2.963$, $0.079),(\Delta \mu, \mathrm{RMS})=(1.972,0.067)$, and $\left(A_{V}, \mathrm{RMS}\right)=(2.950$, 0.048 ). These results are very close to those obtained in Approach II. The agreement among the results from these three 
Table 2

Spectral Features Deweighted Best-fit Parameters Using Approach II

\begin{tabular}{ccccr}
\hline \hline $\begin{array}{l}\text { SN 2012cu } \\
\text { Phase (days) }\end{array}$ & $E(B-V)$ & $R_{V}$ & $\Delta \mu$ & $\begin{array}{c}A_{V} \\
\left(=R_{V} \cdot E(B-V)\right)\end{array}$ \\
\hline-6.8 & $1.000 \pm 0.002$ & $2.864 \pm 0.014$ & $1.991 \pm 0.009$ & $2.862 \pm 0.010$ \\
-1.7 & $0.998 \pm 0.002$ & $3.012 \pm 0.014$ & $1.912 \pm 0.009$ & $3.006 \pm 0.010$ \\
3.3 & $1.038 \pm 0.002$ & $2.886 \pm 0.013$ & $1.918 \pm 0.008$ & $2.995 \pm 0.009$ \\
6.3 & $1.033 \pm 0.002$ & $2.871 \pm 0.014$ & $1.909 \pm 0.009$ & $2.967 \pm 0.010$ \\
8.3 & $1.019 \pm 0.003$ & $2.917 \pm 0.017$ & $1.913 \pm 0.010$ & $2.972 \pm 0.011$ \\
11.2 & $0.987 \pm 0.003$ & $2.953 \pm 0.018$ & $2.063 \pm 0.009$ & $2.915 \pm 0.011$ \\
16.2 & $0.947 \pm 0.003$ & $3.081 \pm 0.020$ & $2.038 \pm 0.009$ & $2.917 \pm 0.011$ \\
18.2 & $0.953 \pm 0.003$ & $3.078 \pm 0.020$ & $2.008 \pm 0.010$ & $2.933 \pm 0.011$ \\
21.2 & $0.966 \pm 0.004$ & $3.063 \pm 0.019$ & $2.058 \pm 0.009$ & $2.958 \pm 0.010$ \\
23.2 & $0.967 \pm 0.004$ & $2.992 \pm 0.020$ & $2.027 \pm 0.009$ & 1.22 \\
\hline
\end{tabular}

Table 3

Comparison of Approaches I, II, and III

\begin{tabular}{|c|c|c|c|c|c|c|c|c|}
\hline \multirow{2}{*}{ Spectral Mismatch Approach } & \multicolumn{2}{|c|}{$E(B-V)$} & \multicolumn{2}{|c|}{$R_{V}$} & \multicolumn{2}{|c|}{$A_{V}$} & \multicolumn{2}{|c|}{$\Delta \mu$} \\
\hline & Mean & RMS & Mean & RMS & Mean & RMS & Mean & RMS \\
\hline I. Not De-weighted (Section 3.1.1) & 0.983 & 0.046 & 3.035 & 0.131 & 2.973 & 0.051 & 1.952 & 0.068 \\
\hline II. De-weighted (Section 3.1.2) & 1.002 & 0.031 & 2.952 & 0.081 & 2.944 & 0.043 & 1.982 & 0.061 \\
\hline III. Removed (Section 3.1.2) & 0.999 & 0.029 & 2.963 & 0.079 & 2.950 & 0.048 & 1.972 & 0.067 \\
\hline
\end{tabular}

approaches - to well within their RMS fluctuations (Table 3) shows that our best-fit results are not sensitive to the particular scheme of deweighting the regions of high spectral variation, including simply uniformly increasing the measurement uncertainties with no relative deweighting (Approach I). However, the RMS values improve substantially when some reasonable form of deweighting is applied to spectral features.

The average values for the fitting parameters determined by Approach II in this subsection (Section 3.1.2) are adopted as the best-fit host galaxy reddening $(E(B-V))$ and total-toselective extinction ratio $\left(R_{V}\right)$, and distance modulus difference between SN 2012cu and SN $2011 \mathrm{fe}(\Delta \mu)$. This approach gives the lowest RMS on $A_{V}$ and $\Delta \mu$. Our best-fit $E(B-V)$ $(=1.00 \pm 0.03)$ compares well with the value $E(B-V)=$ $0.99 \pm 0.03$ reported by Amanullah et al. (2015) based on broadband photometry from UV to NIR for SN 2012cu using the extinction curve of F99. Our best-fit $R_{V}(=2.95 \pm 0.08)$ is slightly higher than their $R_{V}=2.8 \pm 0.1$. It is striking that optical spectrophotometry alone performs as well as combined UV, optical, and NIR broadband photometry for this case.

For Approach II, the results for the last four epochs are half as far from the mean compared with Approach I. Since late epochs are where light echoes (e.g., Patat 2005) can become most prominent, this is an important improvement.

\subsection{Na I and DIB Features in SN 2012cu Spectra}

We will now examine the NaI and two DIB absorption features in the SN 2012cu spectra. All three features, Na I, DIB at $5780 \AA$, and DIB at $6283 \AA$, are at the same redshift as the $\mathrm{HI}$ along the sightline toward SN 2012cu in NGC 4772 (Haynes et al. 2000).

Na I absorption: for a known dust-to-gas ratio, the $\mathrm{NaI}$ column density can be used to infer the amount of dust along the line of sight. In addition, the correlation between extinction and the EW of Na I was first noticed decades ago (e.g., Merrill \& Wilson 1938) and has been extensively studied since (e.g., Richmond et al. 1994; Turatto et al. 2003; Poznanski et al. 2012; Phillips et al. 2013). We can clearly see the Na I D feature due to the doublet $\lambda \lambda 5896,5890 \AA$ in the SN 2012cu spectra (Figures 8, 9). Sternberg et al. (2014) obtained high resolution measurement of the neutral $\mathrm{Na}$ features of SN 2012cu at four epochs. Their spectrum at a phase of 8 days gives $\left(\mathrm{EW}_{\mathrm{D} 1}, \mathrm{EW}_{\mathrm{D} 2}\right)=(857 \pm 6 \mathrm{m \AA}, 928 \pm 6 \mathrm{m \AA})$, and shows that the lines are so saturated that the Na I column density cannot be measured accurately.

We measure EW(NaD) in our low resolution SNIFS spectra for 16 epochs, spanning phases of -6.8 days to 46.2 days, for which the $\mathrm{S} / \mathrm{N}$ was adequate. Our model consisted of Gaussians for the D1 and D2 components, simultaneously fit with a background parameterized by a fourth order polynomial. The widths of the Gaussians are set equal to the $3.42 \mathrm{~A}$ RMS of the SNIFS line spread function. The ratio of the lines was set to unity to reflect the strong saturation observed in the UVES spectrum. The SNIFS spectrum taken at the same phase as the UVES spectrum yields $\mathrm{EW}(\mathrm{NaD})=1790 \pm 80 \mathrm{~m} \AA$ in good agreement with the UVES results $\mathrm{EW}_{\mathrm{D} 1}+\mathrm{EW}_{\mathrm{D} 2}=1785 \pm$ $8 \mathrm{~mA}$. For the full SNIFS spectral time series, we find a mean of $\langle\mathrm{EW}(\mathrm{NaD})\rangle=1560 \mathrm{m \AA}$ with $\mathrm{RMS}=130 \mathrm{m \AA}$, with $\chi_{\nu}^{2}=3.0$. This EW is marginally lower than the values from the UVES spectrum. But the somewhat high $\chi_{\nu}^{2}$ suggests the presence of extra noise, likely due to the residual structure in $\mathrm{SN}$ spectral features that is difficult to completely remove from our low-resolution spectra. There is no indication of any coherent trend with phase. Because the Na D line is so strongly saturated, only a variation having a velocity at the extremities of the lines could have been detected.

DIB features: the DIBs have a well-known association with dust (e.g., Heger 1922; Merrill 1934; Herbig 1995; Cox \& Patat 2008; Hobbs et al. 2008). We find many DIB features in the high resolution VLT UVES spectrum of SN 2012cu at a phase of 8 days. The DIBs at $5780 \AA$ and $6283 \AA$ are strong enough to be found in the SNIFS spectra (Figures 8, 9). Phillips 


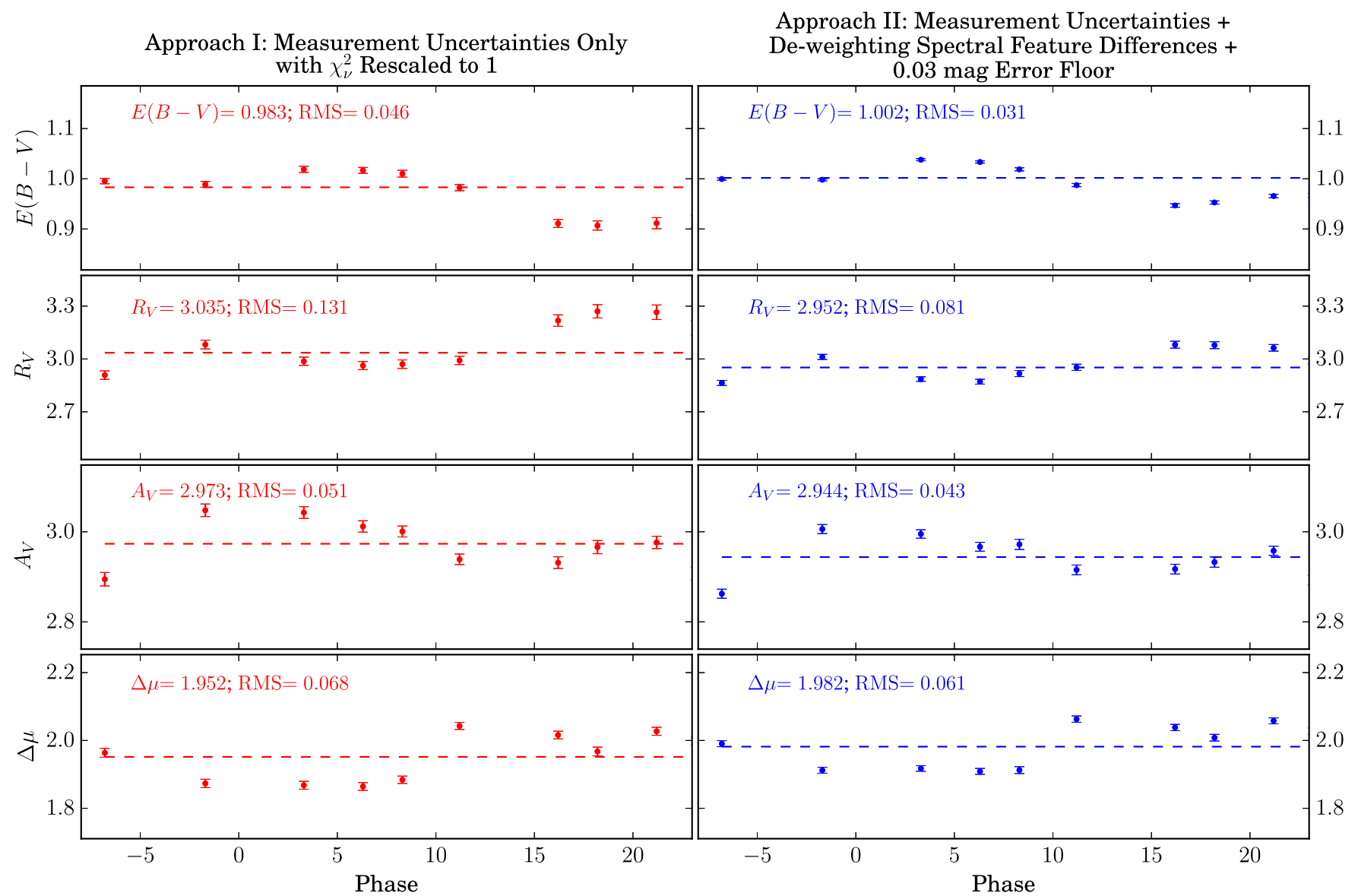

Figure 7. Best-fit $R_{V}, E(B-V), \Delta \mu$, and the derived quantity, $A_{V}$, for the 10 phases of SN 2012cu between -6.8 and 23.2 days. The weighted averages of these quantities are drawn as dashed lines and are printed in their respective panels, along with the RMS dispersions. Left column: summary of best-fit results from Approach I, where we only take into account measurement uncertainties, but rescale to $\chi_{\nu}^{2} \sim 1$ (see the text) to determine the uncertainties of the fitting parameters for each phase. Right column: summary of best-fit results from Approach II, where in addition to taking into account the measurement uncertainties, we deweight the wavelength regions where the spectral differences are high (see the text) and add a 0.03 mag error floor. The results from these two approaches differ slightly and only past day 15. Overall, Approach II more than halves the variance for $R_{V}$. The anti-correlation across the phases between $R_{V}$ and $E(B-V)$ can be seen in both cases. The RMS scatters seen here (and in Tables 1 and 2) are larger than what the per-phase error bars indicate. We address this discrepancy in Section 4.2.

et al. (2013) used the EW of the DIB feature at $5780 \AA$ to measure the extinction for a number of SNe Ia. We find this feature in our spectra for phases $-6.8,-3.8,-1.7,3.3$, and 6.3 days (Figure 9). This feature is located in a Si II absorption line, making it challenging to fit. The approach that works best is to simultaneously fit this absorption feature and the background in a $100 \AA$ window around $5780 \AA$ with a Gaussian and a third order polynomial (Figure 10). The convolution of this feature, with an intrinsic FWHM of $2.11 \AA$ (Hobbs et al. 2008; Phillips et al. 2013), and the SNIFS line-spread-function for the red channel yields a net RMS width of $3.54 \AA$. We thus fix the width of the Gaussian at this value. (From the UVES spectrum we also identify the weak $5800 \AA$ DIB, of which we can see a hint in the panel for phase -3.8 days in Figure 10. At the existing $\mathrm{S} / \mathrm{N}$, it does not affect our fit.) After normalizing by the fitted background, we find the values $\operatorname{EW}_{\text {DIB }}(5780 \AA)=$ $292 \pm 97,390 \pm 76,355 \pm 62,370 \pm 74$, and $432 \pm 71 \mathrm{m \AA}$ for these five phases, respectively. The weighted mean is $357 \pm 34 \mathrm{m \AA}$, and there is no evidence of time variation. From the high resolution VLT UVES spectrum at 8 days we measure $\mathrm{EW}_{\text {DIB }}(5780 \AA)=360 \pm 5 \mathrm{~m} \AA$, in agreement with the results from the lower resolution SNIFS spectra.

\section{Discussion}

We now discuss some of the implications of our measurements, specifically, the distance to the SN 2012cu host galaxy and the nature of the veiling dust.

\subsection{Measurement of Host Distance}

The distance modulus differences between SN 2012cu and SN 2011 fe $(\Delta \mu)$ from Approach I (Section 3.1.1), II, and III (both in Section 3.1.2) are in agreement with each other- to better than their respective RMS dispersions (Table 3). As presented in Section 3.1.2, the average value over 10 epochs from Approach II has been adopted as the best-fit relative distance modulus: $(\Delta \mu, \mathrm{RMS})=(1.982,0.061) \mathrm{mag}$. If we combine the gray scatter for SN 2012cu and SN 2011fe mentioned in Section 3.1.1, we expect an RMS across the phases to be 0.052 mag. With 10 phases, we expect the uncertainty on the RMS to be 0.013 mag. Thus, it is likely that the gray scatter can fully explain the RMS of $0.061 \mathrm{mag}$ for $\Delta \mu$. But it is also possible that there are additional sources of uncertainty that may not be statistical. Below we consider both possibilities.

The distance to the host galaxy of SN 2011fe, M101, has been measured most recently and accurately using the Cepheid 


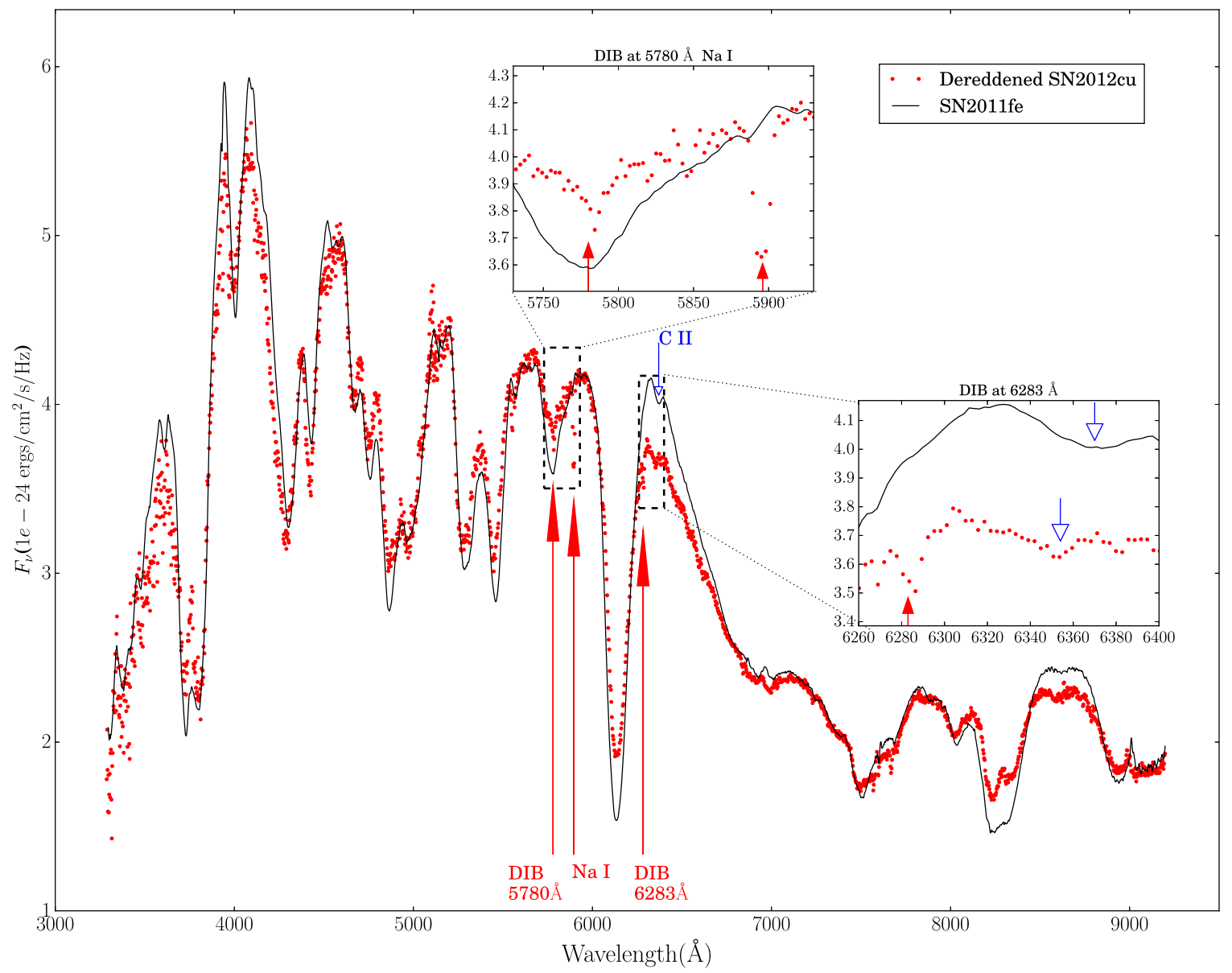

Figure 8. Na I and DIB features. The solid line (black) and the dots (red) show the spectra of SN $2011 \mathrm{fe}$ at -2.0 days and SN $2012 \mathrm{cu}$ at -1.7 days, respectively. The spectrum of SN 2012cu has been dereddened according to the best-fit $E(B-V)$ and $R_{V}$ (see Figure 6 or Table 2) for this phase. Its flux has also been scaled according to the best-fit $\Delta \mu$ to correspond to the distance of SN 2011 fe. The DIB absorption at $5780 \AA$ and the Na I feature can be clearly seen (red arrows with filled arrowheads). We also indicate another known DIB feature at $6283 \AA$ (red arrow with filled arrowhead). All three features are at the redshift of the $\mathrm{H}$ I toward SN2012cu in NGC 4772 (Haynes et al. 2000). The intrinsic C II $\lambda 6580 \AA$ feature is also shown (blue arrow with unfilled arrowhead).

period-luminosity relation and "tip of the red giant branch" (TRGB) techniques. Adjusting the published values from Shappee \& Stanek (2011), Mager et al. (2013), Nataf (2015), and Lee \& Jang (2012) to a common distance modulus of $18.477 \pm 0.033 \mathrm{mag}$ for the LMC (Freedman et al. 2012) gives a distance modulus to M101 of $29.114 \pm 0.072 \mathrm{mag}$. The RMS scatter between the five measurements included is $0.10 \mathrm{mag}$ and the weighted means of the Cepheid and TRGB measurements agree within 0.04 mag. Adopting this distance to M101 with our measured $\Delta \mu$ results in a distance modulus of $31.11 \pm 0.15 \mathrm{mag}$ for NGC 4772, the host of SN 2012cu. This corresponds to $16.6 \pm 1.1 \mathrm{Mpc}$. SNe Ia exhibit a random perobject scatter of $\sim 0.15 \mathrm{mag}$ when standardized using light curve width and color. However, closely matched "twin" SNe Ia like SN 2011fe and SN 2012cu show a much smaller per-object scatter. For the twinness ranking of the pair SN 2012cu and SN 2011fe (at top 10\%), the scatter is expected be $0.086 \mathrm{mag}$ (Fakhouri et al. 2015). Removing the gray scatter of $0.025 \mathrm{mag}$ quoted there, we obtain $0.082 \mathrm{mag}$. If we treat the RMS dispersion for $\Delta \mu$ as purely statistical, the uncertainty on $\Delta \mu$ is $\sigma_{\Delta \mu}=\sqrt{2 \cdot 0.082^{2}+0.061^{2} /(10-1)}=0.12 \mathrm{mag}$. If we take the extreme position that all of the RMS dispersion comes from non-statistical sources, then $\sigma_{\Delta \mu}$ becomes $0.13 \mathrm{mag}$. Thus for the host galaxy of SN 2012cu, after adding the uncertainty for the SN 2011fe distance, $\sigma_{\mu}=0.14$ or $0.15 \mathrm{mag}$, for these two possibilities, respectively.

NGC 4772 is usually considered part of the Virgo Southern Extension (Tully 1982). Using an infall model, Kim et al. (2014) concluded that NGC 4772 is a member of the Virgo Cluster proper. A previous determination of its distance modulus can be found on the Extragalactic Distance Database ${ }^{19}$ (EDD, Tully et al. 2009): $30.96 \pm 0.13 \mathrm{mag}$, corresponding to $15.6 \pm 1 \mathrm{Mpc}$. This is in excellent agreement with our value. Interestingly, the specific distance to NGC 4772 quoted in Tully et al. (2008) is highly discrepant with both the EDD distance, which is the average distance for a group of 11 galaxies, to which NGC 4772 is assigned, and our value. Haynes et al. (2000) found that the stars and the ionized gas in the center of NGC 4772 are counter-rotating, which is often taken as an indication of a merger. If the bulk of the gas in NGC 4772 is from a merger, the H I line width would likely be inflated. For the Tully-Fisher relation, this would result in a measured distance that is too large. Therefore we consider the EDD value, rather than the value from Tully et al. (2008) for this galaxy, as the most appropriate for this comparison.

If, on the other hand, we had mimicked a cosmological fit, e.g., using a value of $\beta \sim 3.1$ (Betoule et al. 2014) and

\footnotetext{
19 http://edd.ifa.hawaii.edu/
} 


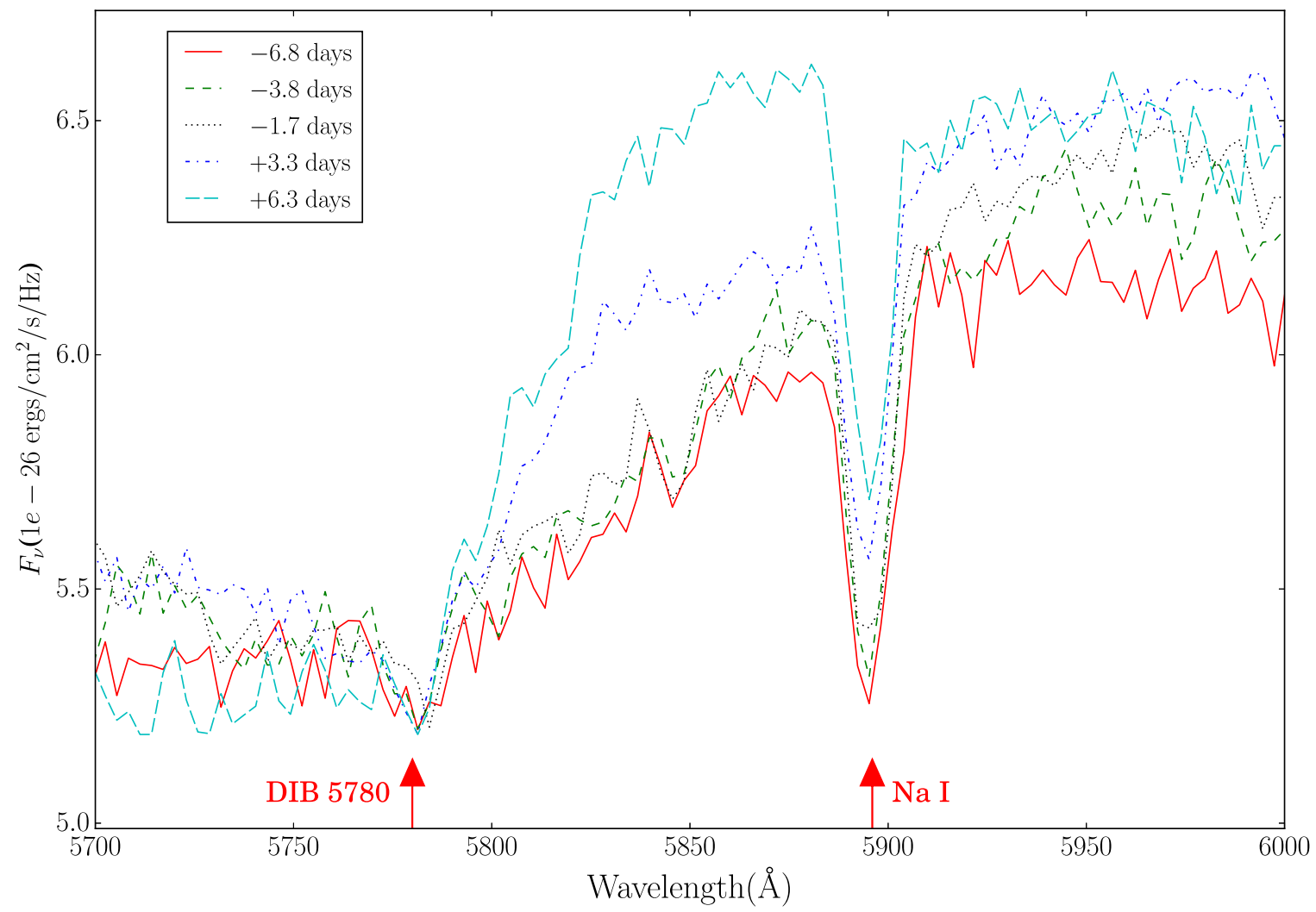

Figure 9. A zoom-in around key ISM features. The solid (red), dashed (green), dotted (black), dashed-dotted (blue), and long-dashed (cyan) lines are SN 2012cu spectra flux-matched at the bottom of a notch near $5780 \AA$ for the phases of $-6.8,-3.8,-1.7,3.3$, and 6.3 days, respectively. The DIB absorption feature at $5780 \AA$, situated near the minimum of a Si II feature, can now be clearly seen. The Na I absorption feature to the right is also readily apparent.

combining it with the SALT $c=0.971$ for SN 2012cu, the estimated distance to NGC 4772 would have been $26 \mathrm{Mpc}$. This would place NGC 4772 well beyond the distance of the Virgo Cluster. Given the uncertainty surrounding the integrity of the H I line width for an apparent merger like NGC 4772, we advocate another independent measurement of the distance, e.g., using the TRGB or the surface brightness fluctuation (SBF) method. If the TRGB or SBF distance also agrees with our distance measurement, that would further validate that our approach of correcting for the extinction using the twins method yields the correct distance.

\subsection{Nature of Dust}

\subsubsection{Upper Limit on Time Variability}

Figure 7 shows that the dispersions for the dust quantities, $R_{V}, E(B-V)$, and $A_{V}$ are small. There is the suggestion of shallow trends in phase for $R_{V}$ and $E(B-V)$, but in opposite directions. The trends appear stronger for Approach I (Figure 7, left column). The effects might be real, but consider the following. 1.) Systematic uncertainties unrelated to dust, e.g., slightly different SN astrophysics even for good but not perfect "twins," likely remain. The (likely small) difference in their time evolution may be responsible for the these trends. The fact that when we deweight the spectral feature differences in Approach II, the trends in $E(B-V)$ and $R_{V}$ clearly become even weaker is consistent with this conjecture. 2.) The remaining very shallow trends in $E(B-V)$ and $R_{V}$ in Approach II (Figure 7, right column) still point in opposite directions. If there were time variation associated with dust, whether due to ISM or CSM, the first order effect would be the time variation of $A_{V}$, with $E(B-V)$ following the same trend (e.g., for ISM, see Patat et al. 2010; Förster et al. 2013; for CSM, see Wang 2005; Goobar 2008; Brown et al. 2015). However, there is no clear trend for $A_{V}$ in Figure 7. While our model does not capture what may be responsible for the very weak trends that remain in $R_{V}$ and $E(B-V)$ in Approach II, their opposite directions may be related to the per-phase anticorrelation between these two quantities noted in Section 3.1.1. We therefore hesitate to claim that the apparent but shallow trends in phase for $R_{V}$ or $E(B-V)$ are real. At this point we regard the RMS values of the fluctuations in $R_{V}$ and $E(B-V)$ as the upper limits for their time variation. To confirm subtler variability would require a pair of $\mathrm{SNe}$ even more closely twinned than SN 2011fe and SN 2012cu. Finally, while it is true that the systematic scatter is greater than the error bars from fitting (Figure 7), the spectral time series for these two $\mathrm{SNe}$ were measured so well that the fitting errors are far below the scale of interest scientifically. The systematic scatters themselves are small. Thus, even if these shallow trends were real, we expect the implications would be minor.

\subsubsection{Lack of Evidence for CSM Dust}

Simulations of extinction due to CSM dust carried out by Wang (2005) and Brown et al. (2015) show that for the phase range probed in this paper, $E(B-V)$ and $A_{V}$ should monotonically decrease over time with a similar fractional rate, and as a result $R_{V}$ would only vary slightly. Those simulated changes in $A_{V}$ and $E(B-V)$ are much greater than 

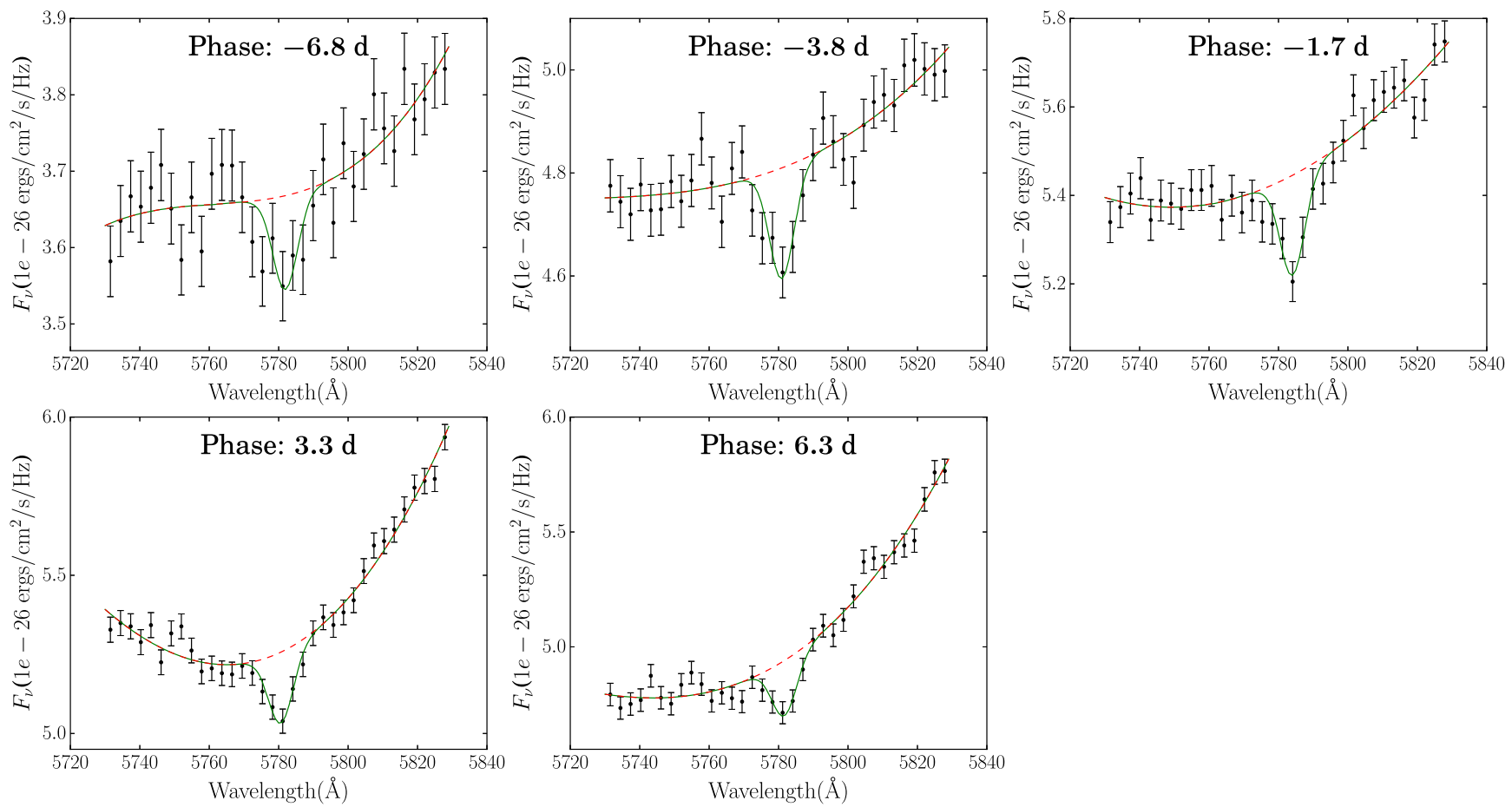

Figure 10. Zoom of the spectral region covering the DIB $5780 \AA$ feature identified in Figure 9 for each epoch where it is well-detected. The green curve in each panel shows a simultaneous fit with a Gaussian, and a third order polynomial for the background (red dashed curve). We normalize by the background and determine the EW for each epoch. We find an average EW of $357 \pm 34 \mathrm{~m} \AA$. This mean value is confirmed by our measurement of $360 \pm 5 \mathrm{~m} \AA$ for the EW of the same feature from a high resolution VLT UVES spectrum at a phase of 8 days. This is about $1 \sigma$ below the MW average, $609_{-242}^{+402} \mathrm{~m} \AA$, expected using our best-fit $A_{V}$ found in Section 3.1.2 (see Phillips et al. 2013).

seen in Figure 7. Changes in CSM ionization conditions due to SN ultraviolet radiation can also lead to variable EW(Na I) (Patat et al. 2007). However, there is no evidence for EW(Na I) variation in Sternberg et al. (2014) or in our measurements of the SNIFS spectra.

\subsubsection{Evidence for ISM Dust}

The $R_{V}$ value we have obtained, $2.952 \pm 0.081$, is very similar to MW average. The expected EW for DIB $5780 \AA$ based on MW observations from Equation (6) of (Phillips et al. 2013) is $609_{-242}^{+406} \mathrm{m \AA}$, and the direct measurement for NGC 4772, $357 \pm 34 \mathrm{m \AA}$, agree within the expected scatter. In addition, as mentioned in Section 3.2, we see many other DIB features in the VLT UVES spectrum. Using the MW average relationship between $\mathrm{EW}(\mathrm{NaI})$ and $E(B-V)$ in Poznanski et al. (2012) and the total EW(Na I) from Sternberg et al. (2014), Amanullah et al. (2015) obtained an $E(B-V)$ prediction that is approximately $2 \sigma$ higher than the measured value for SN 2012cu. To some degree this is expected given the very strong saturation of the $\mathrm{NaI} \mathrm{D}$ absorption and is consistent with the larger scatter on $A_{V}$ versus EW(Na I) found for SNe by Phillips et al. (2013).

Lending support to ISM dust being the cause of the reddening for $\mathrm{SN} 2012 \mathrm{cu}$ is the projected position of SN 2012cu onto a dust lane in its host galaxy (Figure 1). The disk rotation of NGC 4772 was measured by Haynes et al. (2000) who identified an inner ring of $\mathrm{H}$ I that is co-spatial with the dust ring. At the projected location of SN 2012cu, the H I velocity contours of their Figure 12 give a mean of $90 \mathrm{~km} \mathrm{~s}^{-1}$. From the UVES spectrum, we have measured the velocities of the Na I D and DIB $5780 \AA$ extinction tracers to be $89 \mathrm{~km} \mathrm{~s}^{-1}$ and $102 \mathrm{~km} \mathrm{~s}^{-1}$, respectively. These values match well with the $\mathrm{H}$ I velocity at the location of SN 2012cu. Haynes et al. (2000) also provided the bulge-disk decomposition of stellar light, which at the location of SN 2012cu has a bulge-to-disk flux ratio of $\sim 0.07$, indicating that $\mathrm{SN} 2012 \mathrm{cu}$ is most likely in the disk of NGC 4772. Taken together, it is therefore highly likely that SN 2012cu is embedded in or behind the ISM dust lane.

In summary, we find that the observational evidence strongly suggests that the dominant, if not all, foreground dust component in the host of SN 2012cu, is interstellar in nature and is similar to MW dust.

An interesting new avenue for constraining $R_{V}$, independent of SN Ia colors and astrophysics, is the correlation between the apparent dust emissivity power-law index, $\beta_{\mathrm{FIR}}$, and $R_{V}$, found for MW sightlines by Schlafly et al. (2016). It so happens that NGC 4772 was observed in the thermal IR using Herschel, yielding $\beta_{\text {FIR }}=2.1 \pm 0.3$ (Cortese et al. 2014). While typical for many galaxies, this is outside the range spanned by the MW data of Schlafly et al. (2016). But if a linear extrapolation is applicable, it would predict $R_{V}\left(\beta_{\mathrm{FIR}}\right)=2.2 \pm 0.7$. This is lower than, yet, consistent with our measurement based on twin SN Ia optical spectra. $\beta_{\text {FIR }}$ has well-established correlations with galaxy metallicity, gas mass fraction, and stellar mass surface density (Cortese et al. 2014). Unless these differences translate directly into differences in $R_{V}$-a situation that would be relevant for extinction correction of $\mathrm{SNe} \mathrm{Ia}$ - then differences between the properties of the NGC 4772 dust ring and the MW dust could bias this method. The size of the uncertainty further illustrates that it may prove challenging to measure $\beta_{\text {FIR }}$ with sufficient accuracy to help resolve the tension between the cosmologically derived $\beta$ and $\mathrm{SN}$-color based measurements of $R_{V}$. 
It is worth noting that there is at least one other SN Ia with high extinction and a MW-like $R_{V}, \mathrm{SN} 1986 \mathrm{G}$, with $A_{V} \sim 2.0$ and $R_{V}=2.57_{-0.21}^{+0.23}$ (Phillips et al. 2013) based on optical and NIR photometry. ${ }^{20}$ An earlier $R_{V}$ value of 2.4 for SN 1986G was reported by Hough et al. (1987) based on polarimetry and the Serkowski relation between peak polarization wavelength and $R_{V}$ (Serkowski et al. 1975). Even though SN 1986G is considered a weak and peculiar event (Phillips et al. 1987; Branch 1998), the host galaxies for SN $1986 \mathrm{G}$ and SN 2012cu, NGC 5128 and NGC 4772, respectively, are both large, mostly passive galaxies with a "frosting" of young stars and dust lanes indicative of a merger (for a review on NGC 5128, see Israel 1998; for NGC 4772, see Haynes et al. 2000). Like SN 2012cu, SN 1986G is projected onto its host galaxy dust lane and the dust lane is considered to be the source of its reddening (Phillips et al. 1987, 2013).

\section{Conclusions}

We have used a high-quality spectrophometric time series obtained by the SNfactory to study the highly reddened Type Ia SN 2012cu. We analyzed 10 phases between -6.8 days and 23.2 days, using the phase-matched spectra of SN 2011fe as an unreddened template. By simultaneously fitting for $E(B-V)$, $R_{V}$, and $\Delta \mu$, the distance modulus difference between the two $\mathrm{SNe}$, we have found that SN 2012cu is highly reddened, with $(E(B-V), \mathrm{RMS})=(1.002,0.031),\left(R_{V}, \mathrm{RMS}\right)=(2.952$, $0.081),\left(A_{V}, \mathrm{RMS}\right)=(2.944,0.043)$, and $(\Delta \mu, \mathrm{RMS})=(1.982$, $0.061)$. Our best-fit $E(B-V)$ agrees well with the corresponding value reported in Amanullah et al. (2015) for SN 2012cu based on broadband photometry from UV to NIR, and our bestfit $R_{V}$ is slightly higher than theirs.

Spectral Diversity and Relative Distance Measurement with Supernova Twins. We find that SN 2012cu and SN 2011fe are excellent spectroscopic twins according to the method of Fakhouri et al. (2015). We have treated the modest spectral differences that remain in different ways. The consistency of our measurement of $A_{V}$ and $\Delta \mu$ between these approaches demonstrates that despite the presence of spectral feature differences, spectroscopically twinned $\mathrm{SNe}$ can be used to accurately measure relative distances, in this case between M101 and NGC 4772, the host galaxies to the two SNe, to $6.0 \%$.

Host Distance. We measure the distance modulus to the host galaxy of SN 2012cu, NGC 4772, to be $31.10 \pm 0.15 \mathrm{mag}$, corresponding to a distance of $16.6 \pm 1.1 \mathrm{Mpc}$. Our result is in excellent agreement with the value found on EDD.

Nature of Dust Toward SN 2012cu. While it is difficult to completely eliminate a contribution from circumstellar dust, together the following factors strongly suggest that the dominant dust component along the line of sight to SN 2012cu is interstellar in nature and is similar to MW dust:

(1) it is highly likely that SN 2012cu is embedded in or behind its host-galaxy dust lane;

(2) the agreement between our best-fit $R_{V}$ and the MW average value;

(3) the lack of time variation in $E(B-V)$ and $A_{V}$;

(4) the lack of time varying Na I (or DIB $5780 \AA$ ) absorption;

\footnotetext{
${ }^{20}$ Even though Phillips et al. (2013) used the extinction curve of CCM89, their $R_{V}$ value for SN $1986 \mathrm{G}$ might not be severely underestimated due to the dilution of the 5500-8900 А region by their inclusion of NIR photometry (see Appendix A).
}

(5) our measurement of the $5780 \AA$ DIB and $A_{V}$ agree with the average MW relation;

(6) the presence of many other DIB features identified in the high resolution VLT UVES spectrum;

(7) and our measurements of Na I absorption and $A_{V}$ agree with the broad trend seen in the MW.

We thank the technical staff of the University of Hawaii $2.2 \mathrm{~m}$ telescope, and Dan Birchall for his observing assistance. We recognize the significant cultural role of Maunakea within the indigenous Hawaiian community, and we appreciate the opportunity to conduct observations from this revered site. This work was supported in part by the Director, Office of Science, Office of High Energy Physics of the U.S. Department of Energy under Contract No. DE-AC02-05CH11231. We thank the Gordon \& Betty Moore Foundation for their continuing support. X.H. acknowledges the University of San Francisco (USF) Faculty Development Fund. Z.R. was supported in part by a USF Summer Undergraduate Research Fellowship. Support in France was provided by CNRS/ IN2P3, CNRS/INSU, and PNC; L.P.N.H.E. acknowledges support from LABEX ILP, supported by French state funds managed by the ANR within the Investissements d'Avenir programme under reference ANR-11-IDEX-0004-02. N.C. is grateful to the LABEX Lyon Institute of Origins (ANR-10LABX-0066) of the Universite de Lyon for its financial support within the program "Investissements d'Avenir" (ANR-11-IDEX0007) of the French government operated by the National Research Agency (ANR). Support in Germany was provided by the DFG through TRR33 "The Dark Universe;" and in China from Tsinghua University 985 grant and NSFC grant No 11173017. N. S. acknowledges the support from World Premier International Research Center Initiative (WPI), MEXT, Japan, and JSPS Program for Advancing Strategic International Networks to Accelerate the Circulation of Talented Researchers. This work is partially based on observations made with ESO Telescopes at the La Silla Paranal Observatory, Chile, under program 289.D-5035. Some results were obtained using resources and support from the National Energy Research Scientific Computing Center, supported by the Director, Office of Science, Office of Advanced Scientific Computing Research of the U.S. Department of Energy under Contract No. DE-AC02-05CH11231.

\section{Appendix A \\ Comparison of Three Extinction Curve Models}

Here we compare the extinction curve models of CCM89, OD94, and F99 for our application. We choose the SN 2012cu phase of 11.2 days (pair \#6 in Table 1), for which the spectral features of SN 2011fe and SN 2012cu are the most similar. Examination of several phases indicates that the conclusions presented below are independent of the phase chosen. To provide an initial assessment of these issues, we first use the best-fit $E(B-V), R_{V}$, and $\Delta \mu$ found for F99 in Section 3.1.1 to deredden SN 2012cu with each of these three extinction curves and compare them to the SN 2011fe spectrum at 11.0 days. We present the results in Figure 11. For OD94 and CCM89, while the fits are virtually indistinguishable from F99 for wavelengths blueward of $5200 \AA$, there clearly is tension for these two curves with the data in the wavelength region of 5500-8900 $\AA$. This region roughly corresponds to the $r$ and $i$ bands, and the tension between extinction curves here was first pointed out by F99. For MW sight lines, when $R_{V}=3.1$ is used for CCM89 and F99, they show that CCM89 


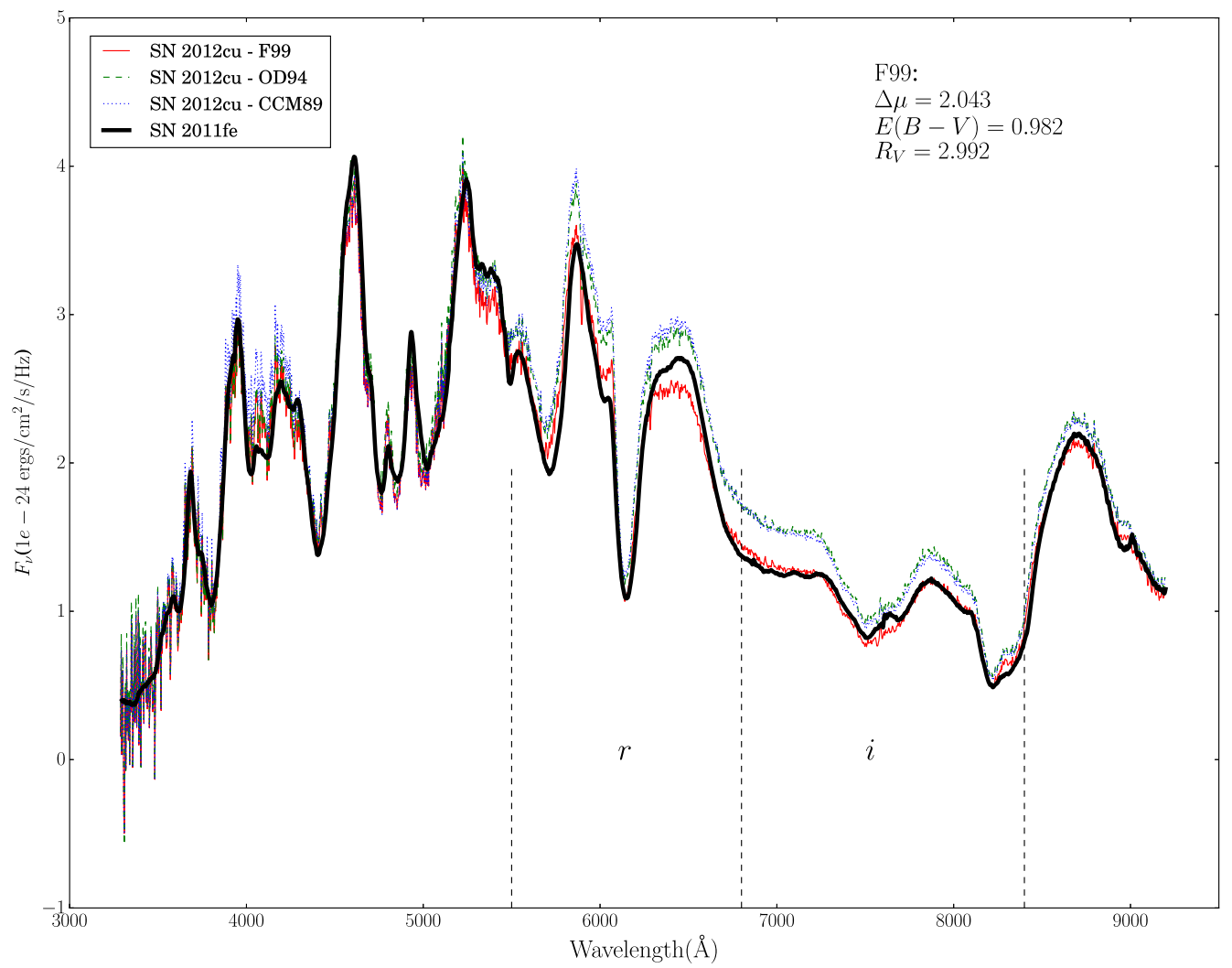

Figure 11. Using the best-fit $E(B-V), R_{V}$, and $\Delta \mu$ for the F99 extinction curve model by Approach I in Section 3.1.1 (see Table 2), the spectrum of SN 2012cu at 11.2 days is dereddened with the F99 (red line), OD94 (green dashed line), and CCM89 extinction curve models (blue dotted line), and then adjusted by $\Delta \mu$ to match the brightness of SN $2011 \mathrm{fe}$. Also plotted is the spectrum of SN $2011 \mathrm{fe}$ at 11.0 days (thick black line), used as the template. The vertical dashed lines demarcate, roughly, the boundaries of the $r$ and $i$ filters.

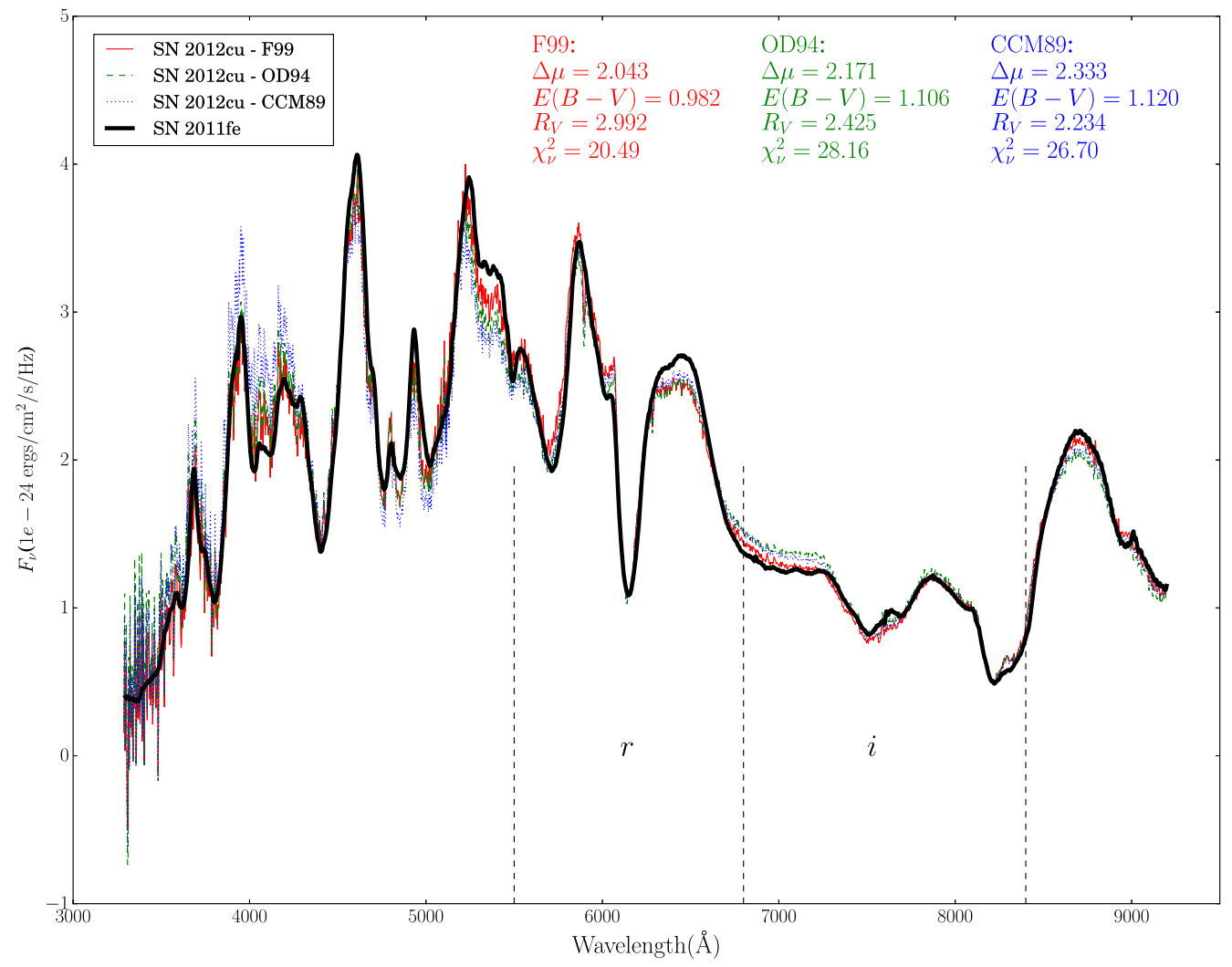

Figure 12. Comparison of the best-fit $E(B-V), R_{V}$, and $\Delta \mu$ found for each of the three extinction curve models, using Approach I in Section 3.1.1. The spectrum of SN $2012 \mathrm{cu}$ at 11.2 days is dereddened with F99 (red line), OD94 (green dashed line), and CCM89 (blue dotted line), adjusted according to $\Delta \mu$ to match the brightness of SN $2011 \mathrm{fe}$. Also plotted is the spectrum of SN $2011 \mathrm{fe}$ at 11.0 days (thick black line), used as the template. The vertical dashed lines indicate the boundaries of the $r$ and $i$ filters. 


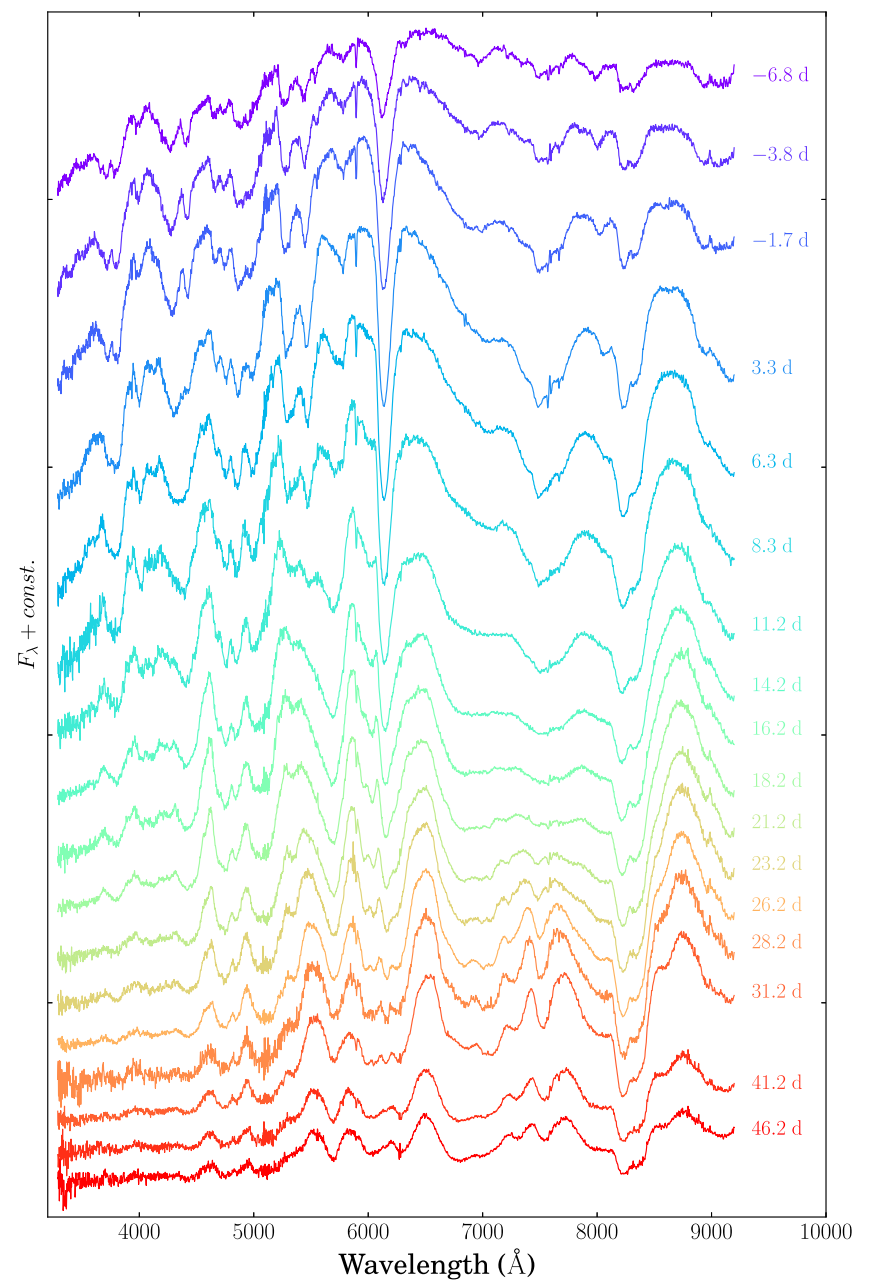

Figure 13. SNIFS spectrophotometric time series of SN 2012cu for phases of -6.8 days to 46.2 days.

overcorrects in the wavelength range that corresponds to the $r$ and $i$ bands (their Figure 6).

Figure 12 shows that this problem with CCM89 and OD94 persists even when $E(B-V), R_{V}$, and $\Delta \mu$ are optimized for each extinction curve model individually. For this comparison, we used Approach I from Section 3.1.1, where only measurement uncertainties are taken into account, since the deweighting in Approach II has the potential to suppress regions that differ due to the extinction curve rather than $\mathrm{SN}$ features. In this case, the best-fit $R_{V}$ values are (CCM89, OD94, F99) $=(2.23,2.43$, $2.99)$, with corresponding $\chi_{\nu}^{2}=(27,28,20)$. While OD94 and CCM89 now match the data more closely in the wavelength region of $5500-8900 \AA$, clearly their agreement remains inferior to F99. Furthermore, for OD94 and CCM89, the fit in the range 5000-5500 $\AA$ has worsened in a systematic way. That the $R_{V}$ values for CCM89 and OD94 are now lower is not a surprise; given the tension seen in Figure 11, CCM89 and OD94 need to be "steeper" to fit the the wavelength range of $5500-8900 \AA$ better, which translates to a lower best-fit $R_{V}$.

In both comparison approaches, F99 clearly provides the best fit, and OD94 provides the worst. This finding is in agreement with Schlafly et al. (2010), who compared the predictions of the same three extinction curve models with the colors of stars from the Sloan Digital Sky Survey (SDSS) and found that F99 fit the data the best, and OD94 fit the poorest. The extinction curves of OD94, and to a lesser extent, of CCM89, were disfavored because they underpredicted the difference between the $r-i$ and $i-z$ colors (see Figure 18 of Schlafly et al. 2010). These correspond to the same wavelength regions where we have found tension for OD94 and CCM89. Berry et al. (2012) compared the predictions of the same three extinction curves with stellar photometry from SDSS, and they also found OD94 to be inconsistent with data, although they considered CCM89 to be acceptable.

In addition, using extragalactic sources to constrain the MW dust properties, Mörtsell (2013) compared F99 and CCM89. Though their analysis was based on colors, it is similar to ours in the sense that both $E(B-V)$ and $R_{V}$ were allowed to vary. They found that the $R_{V}$ values for CCM89 were consistently lower than those for F99. For QSO's, brightest central galaxies, and luminous red galaxies, the $R_{V}$ values were $(\mathrm{CCM} 89, \mathrm{~F} 99)=(3.10 \pm 0.43,3.25 \pm 0.37),(2.89 \pm 0.88$, $3.42 \pm 0.48)$, and $(1.95 \pm 0.35,3.16 \pm 0.36)$, respectively, where the uncertainties correspond to their $95.4 \%$ confidence level. Our results, as presented in Figure 12, show the same pattern. They also made a direct comparison between the extinction values of CCM89 and F99 for the same $R_{V}$. From their Figure 13, it is clear that for $R_{V}=3$, the extinction correction $\left(A_{\lambda}\right)$ is less for F99 than for CCM89 in the wavelength region of the $r$ and $i$ bands. This is exactly as F99 pointed out and as we have shown in our Figure 11.

Amanullah et al. (2015) reported that for SN 2012cu their best-fit $R_{V}$ for F99 $(2.8 \pm 0.1)$ and OD94 $(2.8 \pm 0.2)$ are in agreement. This may be due to the fact that the wavelength coverage in their analysis was from UV to NIR. The wide wavelength coverage may have diluted the discrepancy in the wavelength range between 5500 and $8900 \AA$. However, the uncertainty for their OD94 best-fit $R_{V}$ is twice as large as that for F99. We suspect that the larger uncertainty and higher $\chi_{\nu}^{2}$ are due to the discrepancy between extinction curve models in the $r$ and $i$ wavelength range.

\section{Appendix B SN 2012cu Spectral Time Series Data}

Observations of SN 2012cu were obtained by the SNfactory with its SuperNova Integral Field Spectrograph (SNIFS). SNIFS is a fully integrated instrument optimized for automated observation of point sources on a structured background over the full ground-based optical window at moderate spectral resolution. It consists of a high-throughput wide-band purelenslet integral field spectrograph (IFS; Bacon et al. 1995, 2001), a parallel photometric channel to image the stars in the vicinity of the IFS field of view to monitor atmospheric transmission during spectroscopic exposures, and an acquisition/guiding channel. The IFS possesses a fully filled 6". $4 \times 6$ ". 4 spectroscopic field of view subdivided into a grid of $15^{\prime \prime} \times 15^{\prime \prime}$ spatial elements, a dual-channel spectrograph covering 3200-5200 A and 5100-10,000 $\AA$ simultaneously, and an internal calibration unit (continuum and arc lamps). SNIFS is continuously mounted on the south bent Cassegrain port of the University of Hawaii $2.2 \mathrm{~m}$ telescope on Maunakea. The telescope and instrument, under script control, are operated remotely.

SNfactory follow-up observations of SN 2012cu commenced on 2012 June 20, and span 53 nights. The observing $\log$ is presented in Table 4 . The nominal observational cadence of 2-3 nights was maintained until 31.4 days after maximum brightness. The SN was observed for two more nights. The last spectra reported here were obtained 2012 August 12. All 
Table 4

Observation Log

\begin{tabular}{|c|c|c|c|c|c|c|c|}
\hline Phase (days) & UTC Date & MJD & Photometric & Standard Stars ${ }^{\mathrm{a}}$ & Exp. Time(s) & Airmass & Seeing $\left({ }^{\prime \prime}\right)$ \\
\hline-6.8 & 2012 Jun 20.3 & 56098.3 & $\mathrm{~N}$ & 14 & 820.0 & 1.26 & 0.81 \\
\hline-3.8 & 2012 Jun 23.3 & 56101.3 & $\mathrm{Y}$ & 10 & 920.0 & 1.16 & 1.00 \\
\hline-1.7 & 2012 Jun 25.4 & 56103.4 & $\mathrm{Y}$ & 11 & 1620.0 & 1.81 & 1.82 \\
\hline 3.3 & 2012 Jun 30.3 & 56108.3 & Y & 12 & 2440.0 & 1.65 & 1.65 \\
\hline 6.3 & 2012 Jul 3.3 & 56111.3 & $\mathrm{Y}$ & 8 & 1220.0 & 1.62 & 1.03 \\
\hline 8.3 & $2012 \mathrm{Jul} 5.3$ & 56113.3 & $\mathrm{Y}$ & 13 & 920.0 & 1.64 & 1.95 \\
\hline 11.2 & 2012 Jul 8.3 & 56116.3 & $\mathrm{Y}$ & 19 & 1020.0 & 1.44 & 2.06 \\
\hline 14.2 & 2012 Jul 11.3 & 56119.3 & $\mathrm{~N}$ & 12 & 1020.0 & 1.53 & 1.09 \\
\hline 16.2 & $2012 \mathrm{Jul} 13.3$ & 56121.3 & $\mathrm{~N}$ & 10 & 920.0 & 1.69 & 1.31 \\
\hline 18.2 & $2012 \mathrm{Jul} 15.3$ & 56123.3 & $\mathrm{Y}$ & 10 & 1520.0 & 1.74 & 1.27 \\
\hline 21.2 & 2012 Jul 18.3 & 56126.3 & $\mathrm{Y}$ & 11 & 2740.0 & 1.66 & 2.16 \\
\hline 23.2 & 2012 Jul 20.3 & 56128.3 & $\mathrm{Y}$ & 8 & 920.0 & 1.76 & 1.31 \\
\hline 26.2 & 2012 Jul 23.3 & 56131.3 & $\mathrm{~N}$ & 13 & 1520.0 & 1.62 & 1.31 \\
\hline 28.2 & 2012 Jul 25.3 & 56133.3 & Y & 11 & 320.0 & 1.50 & 1.17 \\
\hline 31.2 & 2012 Jul 28.3 & 56136.3 & $\mathrm{Y}$ & 5 & 1220.0 & 1.75 & 1.23 \\
\hline 41.2 & 2012 Aug 7.3 & 56146.3 & Y & 9 & 820.0 & 2.07 & 1.37 \\
\hline 46.2 & 2012 Aug 12.3 & 56151.3 & $\mathrm{Y}$ & 12 & 820.0 & 2.33 & 1.46 \\
\hline
\end{tabular}

Note.

${ }^{a}$ Number of standard stars observed during the night and used for atmospheric extinction and telluric absorption correction.

spectra were reduced using SNfactory's dedicated data reduction pipeline (Bacon et al. 2001; Aldering et al. 2006; Scalzo et al. 2010). For non-photometric nights, we correct for clouds using field stars in the SNIFS parallel imaging camera. Detailed discussions of the flux calibration and host-galaxy subtraction are provided in Buton et al. (2013) and Bongard et al. (2011), respectively. The final spectrophotometric time series of SN 2012cu in the observer's frame is displayed in Figure 13. A tar file containing these spectra is available at http://snfactory.lbl.gov/TBD.

\section{References}

Aldering, G., Adam, G., Antilogus, P., et al. 2002, Proc. SPIE, 4836, 61 Aldering, G., Antilogus, P., Bailey, S., et al. 2006, ApJ, 650, 510 Amanullah, R., Goobar, A., Johansson, J., et al. 2014, ApJL, 788, L21 Amanullah, R., Johansson, J., Goobar, A., et al. 2015, MNRAS, 453, 3300 Bacon, R., Adam, G., Baranne, A., et al. 1995, A\&AS, 113, 347

Bacon, R., Copin, Y., Monnet, G., et al. 2001, MNRAS, 326, 23 Barone-Nugent, R. L., Lidman, C., Wyithe, J. S. B., et al. 2012, MNRAS, 425, 1007

Berry, M., Ivezić, Ž., Sesar, B., et al. 2012, ApJ, 757, 166

Bessell, M., \& Murphy, S. 2012, PASP, 124, 140

Betoule, M., Kessler, R., Guy, J., et al. 2014, A\&A, 568, A22

Bianchi, L., Conti, A., \& Shiao, B. 2014, AsSpR, 53, 900

Bongard, S., Soulez, F., Thiébaut, É., \& Pecontal, É. 2011, MNRAS, 418, 258

Branch, D. 1998, ARA\&A, 36, 17

Brown, P. J., Smitka, M. T., Wang, L., et al. 2015, ApJ, 805, 74

Buton, C., Copin, Y., Aldering, G., et al. 2013, A\&A, 549, A8

Cardelli, J. A., Clayton, G. C., \& Mathis, J. S. 1989, ApJ, 345, 245

Chotard, N., Gangler, E., Aldering, G., et al. 2011, A\&A, 529, L4

Cortese, L., Fritz, J., Bianchi, S., et al. 2014, MNRAS, 440, 942

Cox, N. L. J., \& Patat, F. 2008, A\&A, 485, L9

de Vaucouleurs, G., de Vaucouleurs, A., Corwin, H. G., Jr., et al. 1991, Third Reference Catalogue of Bright Galaxies, Vol. I, Vol. II, Vol. III (New York: Springer)

Draine, B. T. 2003, ARA\&A, 41, 241

Fakhouri, H. K., Boone, K., Aldering, G., et al. 2015, ApJ, 815, 58 Fitzpatrick, E. L. 1999, PASP, 111, 63

Foley, R. J., Fox, O. D., McCully, C., et al. 2014, MNRAS, 443, 2887 Foley, R. J., \& Kasen, D. 2011, ApJ, 729, 55

Förster, F., González-Gaitán, S., Folatelli, G., \& Morrell, N. 2013, ApJ, 772, 19 Freedman, W. L., Madore, B. F., Scowcroft, V., et al. 2012, ApJ, 758, 24 Ganeshalingam, M., Cenko, S. B., Li, W., et al. 2012, CBET, 3154, 1 Goobar, A. 2008, ApJL, 686, L103
Goobar, A., Johansson, J., Amanullah, R., et al. 2014, ApJL, 784, L12

Guy, J., Astier, P., Baumont, S., et al. 2007, A\&A, 466, 11

Guy, J., Sullivan, M., Conley, A., et al. 2010, A\&A, 523, A7

Haynes, M. P., Jore, K. P., Barrett, E. A., Broeils, A. H., \& Murray, B. M. 2000, AJ, 120, 703

Heger, M. L. 1922, LicOB, 10, 141

Herbig, G. H. 1995, ARA\&A, 33, 19

Hicken, M., Wood-Vasey, W. M., Blondin, S., et al. 2009, ApJ, 700, 1097

Hobbs, L. M., York, D. G., Snow, T. P., et al. 2008, ApJ, 680, 1256

Hough, J. H., Bailey, J. A., Rouse, M. F., \& Whittet, D. C. B. 1987, MNRAS, 227,1

Israel, F. P. 1998, A\&ARv, 8, 237

Itagaki, K., Howerton, S., Noguchi, T., et al. 2012, CBET, 3146, 1

Kelly, P. L., Filippenko, A. V., Burke, D. L., et al. 2015, Sci, 347, 1459

Kim, S., Rey, S.-C., Jerjen, H., et al. 2014, ApJS, 215, 22

Lantz, B., Aldering, G., Antilogus, P., et al. 2004, Proc. SPIE, 5249, 146

Lee, M. G., \& Jang, I. S. 2012, ApJL, 760, L14

Li, A., \& Draine, B. T. 2001, ApJ, 554, 778

Mager, V. A., Madore, B. F., \& Freedman, W. L. 2013, ApJ, 777, 79

Mandel, K. S., Narayan, G., \& Kirshner, R. P. 2011, ApJ, 731, 120

Marion, G. H., Milisavljevic, D., Rines, K., \& Wilhelmy, S. 2012, CBET, 3146, 2

Marion, G. H., Sand, D. J., Hsiao, E. Y., et al. 2015, ApJ, 798, 39

Merrill, P. W. 1934, PASP, 46, 206

Merrill, P. W., \& Wilson, O. C. 1938, ApJ, 87, 9

Mörtsell, E. 2013, A\&A, 550, A80

Nataf, D. M. 2015, MNRAS, 449, 1171

Nugent, P. E., Sullivan, M., Cenko, S. B., et al. 2011, Natur, 480, 344

O'Donnell, J. E. 1994, ApJ, 422, 158

Patat, F. 2005, MNRAS, 357, 1161

Patat, F., Chandra, P., Chevalier, R., et al. 2007, Sci, 317, 924

Patat, F., Cox, N. L. J., Parrent, J., \& Branch, D. 2010, A\&A, 514, A78

Pereira, R., Thomas, R. C., Aldering, G., et al. 2013, A\&A, 554, A27

Perlmutter, S., Aldering, G., Goldhaber, G., et al. 1999, ApJ, 517, 565

Phillips, M. M., Phillips, A. C., Heathcote, S. R., et al. 1987, PASP, 99, 592 Phillips, M. M., Simon, J. D., Morrell, N., et al. 2013, ApJ, 779, 38

Poznanski, D., Prochaska, J. X., \& Bloom, J. S. 2012, MNRAS, 426, 1465

Rest, A., Scolnic, D., Foley, R. J., et al. 2014, ApJ, 795, 44

Richmond, M. W., Treffers, R. R., Filippenko, A. V., et al. 1994, AJ, 107, 1022

Riess, A. G., Filippenko, A. V., Challis, P., et al. 1998, AJ, 116, 1009

Rigault, M., Copin, Y., Aldering, G., et al. 2013, A\&A, 560, A66

Scalzo, R. A., Aldering, G., Antilogus, P., et al. 2010, ApJ, 713, 1073

Schlafly, E. F., \& Finkbeiner, D. P. 2011, ApJ, 737, 103

Schlafly, E. F., Finkbeiner, D. P., Schlegel, D. J., et al. 2010, ApJ, 725, 1175

Schlafly, E. F., Meisner, A. M., Stutz, A. M., et al. 2016, ApJ, 821, 78

Serkowski, K., Mathewson, D. S., \& Ford, V. L. 1975, ApJ, 196, 261

Shappee, B. J., \& Stanek, K. Z. 2011, ApJ, 733, 124

Sternberg, A., Gal-Yam, A., Simon, J. D., et al. 2014, MNRAS, 443, 1849 
Suzuki, N., Rubin, D., Lidman, C., et al. 2012, ApJ, 746, 85

Thomas, R. C., Aldering, G., Antilogus, P., et al. 2011, ApJ, 743, 27

Tripp, R. 1998, A\&A, 331, 815

Tripp, R., \& Branch, D. 1999, ApJ, 525, 209

Tully, R. B. 1982, ApJ, 257, 389

Tully, R. B., Rizzi, L., Shaya, E. J., et al. 2009, AJ, 138, 323

Tully, R. B., Shaya, E. J., Karachentsev, I. D., et al. 2008, ApJ, 676, 184
Turatto, M., Benetti, S., \& Cappellaro, E. 2003, in From Twilight to Highlight: The Physics of Supernovae, ed. W. Hillebrandt \& B. Leibundgut (Berlin: Springer), 200

Wang, L. 2005, ApJL, 635, L33

Wang, X., Filippenko, A. V., Ganeshalingam, M., et al. 2009, ApJL, 699, L139

Wright, E. L., Eisenhardt, P. R. M., Mainzer, A. K., et al. 2010, AJ, 140, 1868

Zhang, T.-M., Lin, M.-Y., \& Wang, X.-F. 2012, CBET, 3146, 3 